\title{
Going beyond Inferior Prefrontal Involvement in Semantic Control: Evidence for the Additional Contribution of Dorsal Angular Gyrus and Posterior Middle Temporal Cortex
}

\author{
Krist A. Noonan ${ }^{1,2}$, Elizabeth Jefferies ${ }^{3}$, Maya Visser $^{2}$, \\ and Matthew A. Lambon Ralph ${ }^{2}$
}

\begin{abstract}
Semantic cognition requires a combination of semantic representations and executive control processes to direct activation in a task- and time-appropriate fashion [Jefferies, E., \& Lambon Ralph, M. A. Semantic impairment in stroke aphasia versus semantic dementia: A case-series comparison. Brain, 129, 2132-2147, 2006]. We undertook a formal meta-analysis to investigate which regions within the large-scale semantic network are specifically associated with the executive component of semantic cognition. Previous studies have described in detail the role of left ventral $\mathrm{pFC}$ in semantic regulation. We examined 53 studies that contrasted semantic tasks with high $>$ low executive requirements to determine whether cortical regions beyond the left $\mathrm{pFC}$ show the same response profile to executive semantic demands. Our findings revealed that right $\mathrm{pFC}$, posterior middle temporal gyrus (pMTG) and dorsal angular gyrus (bordering intraparietal sulcus) were also consistently recruited by executively demanding semantic tasks, demonstrating patterns of activation that were highly similar to the
\end{abstract}

\section{INTRODUCTION}

Semantic cognition requires a combination of semantic representations and executive control processes to direct activation in a task- and time-appropriate fashion. Regardless of the form these experiences take- that is, pictures, words, objects, or environmental sounds-we can readily identify their conceptual significance and use a subset of this information to guide decisions about how we should interact with the outside world (Jefferies \& Lambon Ralph, 2006).

A large constellation of cortical regions support semantic decisions including bilateral anterior temporal lobes (ATLs), pFC, posterior temporal cortex, and angular gyrus (AG; Visser, Jefferies, \& Lambon Ralph, 2010; Binder, Desai, Graves, \& Conant, 2009; Vigneau et al., 2006). Aligning evidence from multiple subfields of cognitive

\footnotetext{
${ }^{1}$ Research Institute for the Care of Older People, Bath, UK,

${ }^{2}$ University of Manchester, ${ }^{3}$ University of York

left ventral pFC. These regions overlap with the lesions in aphasic patients who exhibit multimodal semantic impairment because of impaired regulatory control (semantic aphasia)providing important convergence between functional neuroimaging and neuropsychological studies of semantic cognition. Activation in dorsal angular gyrus and left ventral pFC was consistent across all types of executive semantic manipulation, regardless of whether the task was receptive or expressive, whereas pMTG activation was only observed for manipulation of control demands within receptive tasks. Second, we contrasted executively demanding tasks tapping semantics and phonology. Our findings revealed substantial overlap between the two sets of contrasts within left ventral pFC, suggesting this region underpins domain-general control mechanisms. In contrast, we observed relative specialization for semantic control within pMTG as well as the most ventral aspects of left pFC (BA 47), consistent with our proposal of a distributed network underpinning semantic control.

neuroscience provides an important means of discerning the functional role of each of these neural structures in semantic cognition. At present converging sources of evidence suggests a clear role for certain regions, although the pattern is less clear or contradictory for others. Evidence from semantic dementia, as well as functional neuroimaging and TMS studies of healthy individuals, points to the importance of the bilateral ventrolateral ATLs for the representation of transmodal conceptual knowledge (Visser \& Lambon Ralph, 2011; Binney, Embleton, Jefferies, Parker, \& Lambon Ralph, 2010; Lambon Ralph, Sage, Jones, \& Mayberry, 2010; Mion et al., 2010; Pobric, Jefferies, \& Lambon Ralph, 2010; Patterson, Nestor, \& Rogers, 2007). Similarly, a vast literature of functional neuroimaging studies-supported by TMS and neuropsychological findings-has demonstrated the importance of the left ventral $\mathrm{pFC}$ for the regulation of semantic activation (Hoffman, Jefferies, \& Lambon Ralph, 2010; Whitney, Kirk, O'Sullivan, Lambon Ralph, \& Jefferies, 
2010; Devlin, Matthews, \& Rushworth, 2003; Badre \& Wagner, 2002; Metzler, 2001; Thompson-Schill et al., 1998).

There is greater uncertainty about the functional role of posterior temporal cortex and AG. Functional neuroimaging studies of semantic cognition frequently detect activation in these regions: In a meta-analysis of neuroimaging studies conducted by Binder et al. (2009), these regions showed the highest density of peaks anywhere in the brain. The stroke aphasia literature similarly emphasizes the critical involvement of these regions in language comprehension and semantic processing (Chertkow, Bub, Deaudon, \& Whitehead, 1997; Hart \& Gordon, 1990; Kertesz, Sheppard, \& MacKenzie, 1982; e.g., Turken \& Dronkers, 2011; Stuss \& Alexander, 2007). Damage to specific aspects of temporal and parietal cortex can produce category-specific patterns of impairment (Gainotti, 2000; Warrington \& McCarthy, 1987, 1994; Hillis \& Caramazza, 1991; Warrington \& Shallice, 1984), consistent with the view that specific cortical fields within these regions represent the sensory and motor features of objects (e.g., Martin, 2007). However, contemporary and more historical neuropsychological studies also demonstrate that temporoparietal lesions can give rise to semantic control problems that are highly similar to the deficits associated with pFC damage (Noonan, Jefferies, Corbett, \& Lambon Ralph, 2010; Corbett, Jefferies, Ehsan, \& Lambon Ralph, 2009; Luria, 1976; Head, 1926). It is therefore possible that separate sites within posterior temporal and inferior parietal cortex contribute to semantic representation and control. Given the wealth of semantic-related fMRI studies, the purpose of this study was to undertake a formal metaanalysis of neuroimaging studies that have manipulated executive control demands within the semantic domain to determine whether a network of regions in addition to left ventral pFC—specifically, posterior middle temporal gyrus (pMTG) and dorsal AG (dAG) bordering intraparietal sulcus (IPS)—contribute to the executive regulation of semantic processing.

Multiple lines of evidence already suggest that the neural substrates of semantic control may extend beyond the left ventral pFC. A subset of semantically impaired stroke patients (referred to here as semantic aphasia or SA) present with multimodal semantic impairment. Their lesions are located in left pFC (BA 44, BA 45, BA 47) and/or the temporoparietal region (BA 21, BA 37, BA 39, BA 40; Noonan et al., 2010; Jefferies \& Lambon Ralph, 2006; Luria, 1976; Head, 1926). In contrast to the degradation of semantic representations observed in semantic dementia (which follows from atrophy focused on the anterior temporal region), SA patients retain detailed semantic information but have damage to the neurocognitive systems that regulate and control semantic activation (Jefferies \& Lambon Ralph, 2006). Strikingly, pFC involvement is not required for SA patients to demonstrate impaired semantic control; isolated lesions to temporoparietal cortex produce highly similar impairments in both the verbal and nonverbal domain (Corbett, Jefferies, \& Lambon
Ralph, 2009, 2011). Recently, Schwartz et al. (2009) investigated the neural substrates associated with semantic naming errors in aphasic patients and found a large network of contributing regions including left superior ATL, pMTG, and ventral pFC. Critically, when measures of controlled semantic retrieval and selection were partialled out, only ATL lesions predicted semantic errors, suggesting that pFC and pMTG were primarily involved in executive aspects of semantic processing. These results are supported by evidence from TMS studies in healthy control participants: Stimulation of pMTG and ventral pFC produced an equivalent and selective decrement on executively demanding semantic decisions, with sparing of judgments based on automatic semantic associations (Whitney et al., 2010).

Together these findings fit with the proposal that semantic cognition draws on at least two interactive primary components: First, there is a hub-and-spoke semantic representational system. The "hub" in bilateral ventrolateral ATL extracts modality-invariant conceptual representations from information about the visual, auditory, motor, and verbal attributes of concepts (stored within distributed motor and sensory areas-i.e., the "spokes"; Mayberry, Sage, \& Lambon Ralph, 2011; Lambon Ralph et al., 2010; Pobric et al., 2010; Patterson et al., 2007; Rogers et al., 2004; McClelland \& Rogers, 2003). Recent evidence suggests that the key site for the semantic hub may not be the temporal pole but, instead, is centered on the anterior fusiform (Visser \& Lambon Ralph, 2011; Binney et al., 2010; Mion et al., 2010). Second, semantic control processes (underpinned by interactions within a distributed network including pFC, pMTG, and dAG/ IPS) regulate activation of these representations in a task appropriate and context sensitive fashion (Jefferies, 2013; Noonan et al., 2010; Jefferies \& Lambon Ralph, 2006; Luria, 1976).

Functional neuroimaging research provides considerable support for this framework. However, the main focus of this literature has been concerned with two specific themes: (i) exploring the neural structures responsible for semantic representations and (ii) determining the executive semantic functions of the left ventral pFC, with minimal focus on the role of other cortical regions. With regards to the first point, most meta-analyses of functional neuroimaging studies to date have not explicitly assessed the contribution of semantic control processes to observed patterns of functional activation but have instead focused on aspects of representation structure (Visser et al., 2010; Binder et al., 2009; Gerlach, 2007; Vigneau et al., 2006; Joseph, 2001). Indeed, in the large-scale meta-analysis of verbal semantic processing tasks carried out by Binder et al. (2009), explicit steps were taken to exclude studies and functional contrasts, which varied in their executive processing requirements. However, as several components of semantic control-that is, task-directed retrieval/ selection of memories and integration of these internal representations with external inputs and current goals-are 
an intrinsic part of control-demanding semantic tasks (although not necessarily exclusive to semantic processing), it is difficult to quantify the extent to which patterns of activation in pMTG and subregions of AG may reflect the influence of these processes. The current meta-analysis addresses this issue by adopting the complementary method of directly comparing tasks with high and low semantic control demands, revealing patterns of activation that support more demanding semantic tasks across the brain, in both temporoparietal and prefrontal regions.

Contrasting executively demanding and less demanding semantic conditions has provided much of the groundwork for exploring the functional role of $\mathrm{pFC}$ in semantic cognition. An extensive set of studies have shown that left ventral $\mathrm{pFC}$ plays a key role in regulating semantic activation (Badre \& D'Esposito, 2009; Badre \& Wagner, 2007; Thompson-Schill, 2003; Gold \& Buckner, 2002; Gabrieli, Poldrack, \& Desmond, 1998; Demb et al., 1995). Consequently, understanding the executive semantic parameters, which modulate $\mathrm{pFC}$ activation, provides a means of assessing what tasks/conditions should activate pMTG and dAG/ IPS regions if they are also involved in semantic regulation. To date, a range of tasks have been shown to modulate activation in left ventral pFC. In particular, (i) task structure and (ii) the nature of the stimuli can induce greater requirements for semantic control: Tasks requiring selfdirected retrieval of semantic knowledge or postretrieval selection between competing responses require executive control processes underpinned by left ventral pFC (BA 47/ BA 45). In these studies, it has been shown that the specificity of the judgment (decisions based on global semantic vs. feature-specific similarity) as well as the strength of the semantic association between probe and target (relative to probe and distracter) modulate left ventral pFC activation (Badre, Poldrack, Pare-Blagoev, Insler, \& Wagner, 2005; Wagner, Pare-Blagoev, Clark, \& Poldrack, 2001; Thompson-Schill, D'Esposito, Aguirre, \& Farah, 1997) - that is, a weaker association between probe and target requires a greater degree of on-line exploration of the semantic database. In addition, a number of studies that look at the processing of semantically ambiguous materials - for example, metaphors and homonymshave shown that stimuli that are inherently multifaceted (in terms of their relationship to underlying meaning) also give rise to greater processing demands with the executive semantic system (Hoenig \& Scheef, 2009; Mashal, Faust, Hendler, \& Jung-Beeman, 2009; Chen, Widick, \& Chatterjee, 2008; Gennari, MacDonald, Postle, \& Seidenberg, 2007; Shibata, Abe, Terao, \& Miyamoto, 2007; Stringaris, Medford, Giampietro, Brammer, \& David, 2007; Zempleni, Renken, Hoeks, Hoogduin, \& Stowe, 2007; Lee \& Dapretto, 2006; Rodd, Davis, \& Johnsrude, 2005; Rapp, Leube, Erb, Grodd, \& Kircher, 2004). These findings are unsurprising given that left ventral $\mathrm{pFC}$ has been implicated in a wide range of different executive processes, including task switching, resolution of proactive interference and strategic priming (Gold et al., 2006; Badre \& Wagner, 2005; Brass, Derrfuss, Forstmann, \& Cramon, 2005).

To date, minimal attention has been paid to the finding that a number of the semantically orientated paradigms, which have been used to assess the control properties of left ventral $\mathrm{pFC}$, have also generated activation in pMTG and at the boundary of AG/IPS (Whitney, Jefferies, \& Kircher, 2010b; Zempleni et al., 2007; Gold et al., 2006; Badre et al., 2005; Rodd et al., 2005; Noppeney, Phillips, \& Price, 2004; Thompson-Schill et al., 1997). In parallel, studies of SA patients have revealed that the same kinds of semantic manipulations lead to increasingly poor performance in both temporoparietal and $\mathrm{pFC}$ cases (Noonan et al., 2010; Jefferies \& Lambon Ralph, 2006). Even in the light of these findings, however, the hypothesis that sites within temporoparietal regions are involved in executive semantic processing has rarely been considered in depth in functional neuroimaging studies and, in some cases, the activation peaks-although present in reported summary tables-have not been discussed at all. The current meta-analysis allowed us, therefore, to test the reliability and consistency of the involvement of pMTG and dAG/IPS in semantic control.

The central aim of this study was to assess the degree of convergence between patient and functional neuroimaging-based studies of semantic control in an attempt to elucidate the neurocognitive underpinnings of regulatory processing. Given that previous studies of SA patients have been crucial in highlighting the possible involvement of temporoparietal regions in semantic control, the meta-analysis focused on three specific themes emerging from these neuropsychological studies:

(1) Semantic control is underpinned by a distributed system, which includes posterior middle temporal and $\mathrm{dAG}$ regions, in addition to $\mathrm{pFC}$ : The nature and the degree of semantic impairment in SA patients have been shown to be largely consistent across $\mathrm{pFC}$ and temporoparietal lesion subgroups: Performance is poor on demanding tests of associative semantic knowledge, on assessments with semantically ambiguous materials, on nearest neighbor judgment tasks, which increase the degree of semantic distance between probes and targets and when naming pictures in the context of phonemic miscues, while less intrinsically demanding tests of identity matching (i.e., word-picture matching) are performed relatively well (Corbett, Jefferies, Ehsan, et al., 2009; Corbett et al. 2011; Noonan et al., 2010; Jefferies \& Lambon Ralph, 2006). This suggests that sites within temporoparietal cortex may form part of a large-scale distributed network underpinning semantic control; however, these studies lack spatial precision since SA cases typically have extensive lesions. This meta-analysis examines the role of more focal sites within the large areas of infarct seen in SA patients-specifically the contribution of pMTG and $A G / I P S$. 
(2) Semantic control may be multifaceted: One important yet unresolved issue is whether the sites within the semantic control network - that is, ventral pFC, pMTG dAG/IPS — make differing contributions to semantic control. Patients can show complex patterns of dissociations across tasks that require different types of semantic processing (Kemmerer, Rudrauf, Manzel, \& Tranel, 2012). Moreover, there are some differences in the neuropsychological profiles of SA patients with anterior and posterior lesions. Patients with left pFC lesions have less fluent language production (Berthier, 2001), and they also have greater difficulty than temporoparietal cases in inhibiting previously relevant semantic information-namely, they exhibit "refractory" semantic behavior, that is, declining accuracy in "cyclical" word-picture matching tasks that present a set of semantically related items repeatedly, leading to a build-up of competition between previous and current targets (Gardner et al., 2012; Campanella, Mondani, Skrap, \& Shallice, 2009; Jefferies, Baker, Doran, \& Lambon Ralph, 2007). Similarly, pMTG and dAG/IPS may play distinct roles in the regulation of semantic processing, although these regions do not easily dissociate in studies of brain-injured patients.

(3) Semantic control is amodal and shares neural and cognitive resources with domain-general control: SA patients exhibit deficits on nonverbal semantic processing tasks when assessed with pictures, environmental sounds, and tests of object use, and these studies highlight the same impaired aspects of control as similar explorations with verbal materials (Corbett, Jefferies, Ehsan, et al., 2009; Corbett, Jefferies, et al. 2009; Jefferies \& Lambon Ralph, 2006). Moreover, SA patients exhibit a consistent association between impaired semantic regulation and domain-general control impairments (Jefferies \& Lambon Ralph, 2006; Luria, 1976). The degree of semantic difficulty in SA can be predicted from nonverbal measures of executive control. This fits with a perspective that suggests neural and cognitive resources are shared, to a certain degree, across all forms of executive processing-including verbal semantic, nonverbal semantic and nonsemantic domains (Nagel, Schumacher, Goebel, \& D’Esposito, 2008; Duncan, 2006; Duncan \& Owen, 2000).

However, SA patients have large lesions, which may conceivably encompass regions engaged by both domain-general and more specific aspects of semantic control. Within left pFC, dorsal regions are associated with general executive control, whereas ventral pFC (particularly BA 47) is thought to play a more specific semantic role (Nagel et al., 2008). IPS is also considered to be part of a multidemand network, engaged whenever executive demands are high (Duncan, 2010). Activation within this region has been reported during tasks requiring the top-down selection of specific semantic features (Badre et al., 2005; Thompson-Schill et al., 1997) and during nonsemantic selection (Nagel et al., 2008). pFC and IPS show coupled activation across a wide variety of manipulations of executive control, including interference resolution, response inhibition, attention shifting, and goal-directed cognition (Spreng, Stevens, Chamberlain, Gilmore, \& Schacter, 2010; Nee, Wager, \& Johides, 2007).

The role of posterior temporal cortex and AG require further clarification, since these regions are not classically linked with general executive control. However, recent studies of resting-state functional connectivity suggest that specific regions within both these sites (pMTG and dAG) might form part of a large-scale distributed frontoparietal control network, which flexibly allocates attention to memory representations or external inputs according to task demands and integrates these sources of information about the world (Spreng et al., 2010; Vincent, Kahn, Snyder, Raichle, \& Buckner, 2008). The frontoparietal network comprises large swathes of lateral and anterior pFC, dorsal and anterior parts of AG bordering IPS, and a region of pMTG, which lies anterior to visual area $\mathrm{MT}^{+}$. Semantic judgments about the meanings of words or objects should draw strongly on the frontoparietal network, in addition to semantic representational areas, because they necessarily require integration of external information with internal representations.

\section{METHODS}

Web of Knowledge (www.isiknowledge.com) was used to identify fMRI and PET studies that explored semantic processing using the following search terms: fMRI or PET combined with semantic, or comprebension, or conceptual knowledge. In addition, the following search terms were included to narrow the search to studies looking specifically at semantic control: selection, retrieval, inbibition, control, elaboration, fluency, ambiguity, metaphor, and idiom. The results of these searches were reviewed, and any additional relevant studies known to the authors were included.

The inclusion and the exclusion criteria were as follows: (1) we examined PET and fMRI studies on the topic of executive semantic processing; (2) the studies were published in peer-reviewed journals (in English) between January 1994 and August 2009; (3) studies were included that reported either (i) contrasts that reflected high $>$ low semantic control or (ii) where a task requiring semantic control was contrasted with a equally demanding executive decision in a nonsemantic domain; (4) studies were excluded when they did not report peak coordinates or the reported coordinates were not in standard space (i.e., Talairach or MNI); and (5) studies were excluded if their focus was on patients, gender differences, priming 
without an explicit semantic judgment (e.g., primed lexical decision), bilingualism, development of language/semantics, episodic memory, or sleep consolidation. This search identified $53 \mathrm{fMRI}$ and PET studies (listed in Appendix 1).

Our analyses addressed two broad questions in the literature. First, we investigated which areas are critically important to executive processing in the semantic domain and whether these areas overlap with the distribution of lesions in SA patients. Second, we explored the overlap between brain regions involved in executive processing in the semantic and phonological domains.

To address the first question, we explored the 53 studies that contrasted high $>$ low semantic control (see Appendix 1 for details of contrasts). Where studies included more than one contrast they were included separately if they represented (1) different experiments or (2) the same experiment in a different modality. GingerALE software was used to generate activation likelihood estimations for all voxels in the brain based on the 71 contrasts identified in the literature (395 peaks). For this analysis, and all subsequent ALE analyses, we followed the procedure as described in Laird et al. (2005). Smoothing was implemented with a 10-mm FWHM kernel; the permutation test included 5000 permutations; the false discovery rate (FDR) was set at $\alpha=.05$. To explore the relationship between cortical damage in SA patients and factors affecting cortical activation on executive semantic tasks in functional neuroimaging studies, we defined ROIs from the ALE activation clusters, which overlapped most closely with the maximal areas of damage in SA - that is, left pFC,
AG, and pMTG. Table 1 provides information on these clusters (Clusters 1, 2, 4, and 8). Two clusters in the parietal cortex were detected within close proximity of each other, both with focal peaks in AG (dorsal and mid-AG, respectively; Clusters 4 and 8). These areas were collapsed into a single functional ROI. To determine whether the parameters of semantic tasks differentially contributed toward activation in each ROI, we assessed whether each study/contrast contributed at least one peak to each of the functional clusters. Whether a peak contributed to each functional ROI was determined on the basis of whether the peak fell within the range of maximal coordinates of $x, y$, and $z$ in Talairach space for each ROI, excluding peaks that fell outside the activation cluster. A complementary set of analyses were run using GingerALE (parameters as above) to assess whether differences in the (1) receptive/expressive parameters of semantic tasks and (2) the nature of the executive semantic manipulation within a study influenced the likelihood of activation in pFC, pMTG, and AG.

Studies that compared a semantically demanding task to a phonological task requiring executive processing were used to address the second question. First, GingerALE was used to generate activation likelihood estimation maps for executively demanding tasks relative to low-level baseline or rest conditions. These analyses were performed separately for semantic (eight studies, 80 peaks) and phonological tasks (eight studies, 70 peaks). After identifying commonalities in the executive networks activated by semantic and phonological tasks, we next compared

Table 1. Activation Clusters Derived from the GingerALE Analysis of High > Low Semantic Control Studies

\begin{tabular}{|c|c|c|c|c|c|c|c|c|}
\hline \multirow[b]{2}{*}{ Cluster No. } & \multirow[b]{2}{*}{ Brain Region } & \multirow[b]{2}{*}{ Hemisphere } & \multirow{2}{*}{$\begin{array}{c}\text { Volume } \\
\left(\mathrm{mm}^{3}\right)\end{array}$} & \multicolumn{3}{|c|}{$\begin{array}{c}\text { Talairach } \\
\text { Coordinates }\end{array}$} & \multirow[b]{2}{*}{ Brodmann's Area } & \multirow{2}{*}{$\begin{array}{l}\text { Maximum } \\
\text { ALE Value }\end{array}$} \\
\hline & & & & $x$ & $y$ & $z$ & & \\
\hline 1 & Ventral pFC & Left & 26016 & -45 & 19 & 18 & $45,44,47$ & 0.06 \\
\hline 2 & pMTG & Left & 7232 & -54 & -49 & -2 & 21,37 & 0.03 \\
\hline 3 & Dorsal medial frontal lobe & Left & 5168 & -3 & 18 & 44 & $32,24,8,9$ & 0.03 \\
\hline 4 & AG (dorsal) & Left & 1568 & -41 & -55 & 45 & $39,40,7$ & 0.02 \\
\hline 5 & Ventral pFC & Right & 1304 & 47 & 23 & 26 & $44,45,46$ & 0.02 \\
\hline 6 & Anterior Cingulate & Left & 1056 & -1 & 43 & -1 & 32,10 & 0.02 \\
\hline 7 & Insula cortex & Right & 1000 & 40 & 14 & 14 & - & 0.02 \\
\hline 8 & $\mathrm{AG}$ (mid) & Left & 920 & -39 & -65 & 30 & 39,19 & 0.01 \\
\hline 9 & Ventral pFC & Right & 856 & 35 & 21 & -10 & 47 & 0.02 \\
\hline 10 & Anterior MTG & Left & 800 & -50 & -14 & -14 & 21,20 & 0.02 \\
\hline 11 & Inferior/superior parietal lobe & Right & 576 & 36 & -59 & 39 & 39,7 & 0.01 \\
\hline 12 & Dorsal pFC & Left & 448 & -22 & 49 & 26 & 9,46 & 0.02 \\
\hline 13 & Dorsal pFC & Right & 408 & 37 & 35 & 37 & 9 & 0.02 \\
\hline 14 & Dorsal pFC & Right & 360 & 21 & 40 & 23 & 9 & 0.02 \\
\hline
\end{tabular}


contrasts in the literature, which looked at subtractions of one task from another. For this analysis we included data from 15 studies (63 peaks) that reported semantic $>$ phonological control contrasts and 14 studies that reported the reverse (i.e., phonological $>$ semantic control: 72 peaks). One study reported semantics $>$ phonology but not the reverse contrast.

\section{RESULTS}

\section{The Semantic Control Network}

The results of the first GingerALE analysis revealed a large network of areas that were modulated by executive semantic processing (Table 1). The two largest clusters were in the left pFC and pMTG, respectively (see Figure 1 or Supplementary Figure 1). The pFC cluster had a focal point within BA 45, with a number of additional subpeaks within BA 47 and BA 44. The cluster also extended dorsally into DLPFC. The pMTG cluster had subpeaks in the most posterior portions of the temporal lobe (BA 21/BA 37). Two medial left hemisphere clusters were also detected in and around the anterior cingulate gyrus (BA 32). The larger of the two extended dorsally into (BA 6), whereas the smaller cluster had an anterior extension into frontal polar cortex (BA 10). Two anatomical clusters within close proximity of each other were also detected in AG (BA 39): One had a peak within anterior and $\mathrm{dAG}$, bordering IPS and supramarginal gyrus (SMG); the other was within mid-
AG. In addition to the left hemisphere areas noted above, a number of clusters were detected in the right hemisphere. The largest cluster expanded across dorsal and ventral pFC (BA 44/BA 45/BA 46), and a smaller cluster was also detected in the inferior ventral pFC (BA 47). The results of this analysis suggest that a large-scale distributed network, which includes left pFC, pMTG, and dAG/ IPS, underpins semantic control, consistent with the data from SA patients. This network is highly overlapping with the frontoparietal control network identified by Spreng et al. (2010) and Vincent et al. (2008). Appendix 2 lists the studies that contributed to activation within left pMTG and in/around AG.

\section{Does the Contribution of pFC, MTG, and AG Vary across Different Semantic Tasks?}

In the next set of analyses, we attempted to determine if each of the different types of study in our analysis made equal contributions to each of the anatomical clusters. For these analyses we focused on the three main ROIs highlighted by the investigation of SA patients' lesions (i.e., left pFC, pMTG, and AG). Two main hypotheses exist for the functional roles of these three cortical areas. First, all three areas may make qualitatively similar contributions to semantic control. Second, different areas may be relatively more involved in receptive or expressive tasks or when performing different aspects of semantic control. Our first set of comparisons investigated whether expressive
Figure 1. ALE map for high > low semantic control. Analysis is based on 53 studies (71 contrasts) that compared high $>$ low semantic control Data corrected for multiple comparisons using an FDR threshold of $p<.05$. Only clusters with an extent of $100 \mathrm{~mm}^{3}$ are displayed: (A) left hemisphere, (B) right hemisphere, and (C) medial left hemisphere.

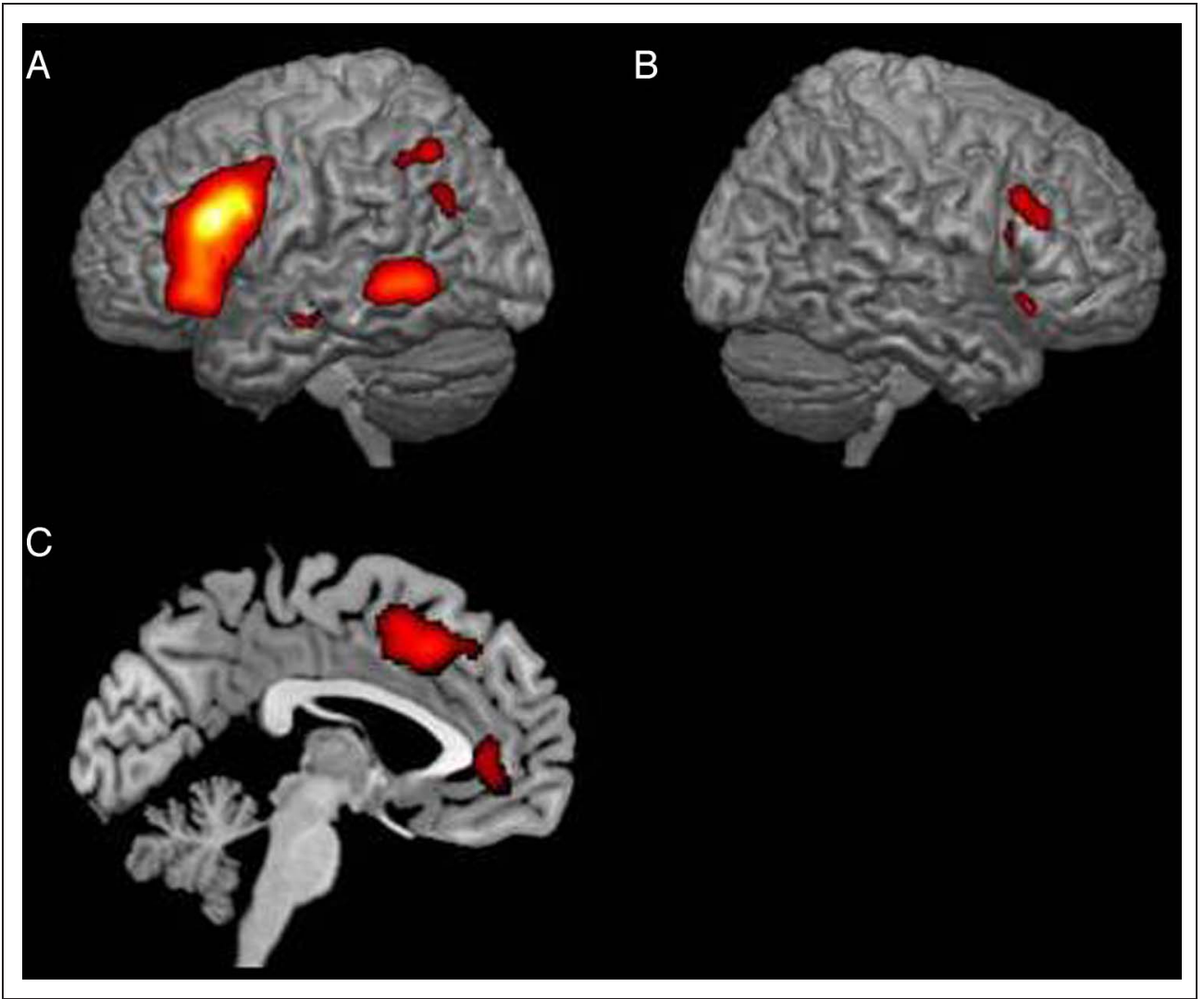


Figure 2. Proportion of studies that contributed a peak to each anatomical clusters of interest for (A) expressive/receptive tasks and (B) different forms of receptive tasks. Figures based on data from 53 studies (71 contrasts). Bars represent the proportion of contrasts in each category, which contributed at least one peak to any of the anatomical clusters of interest.

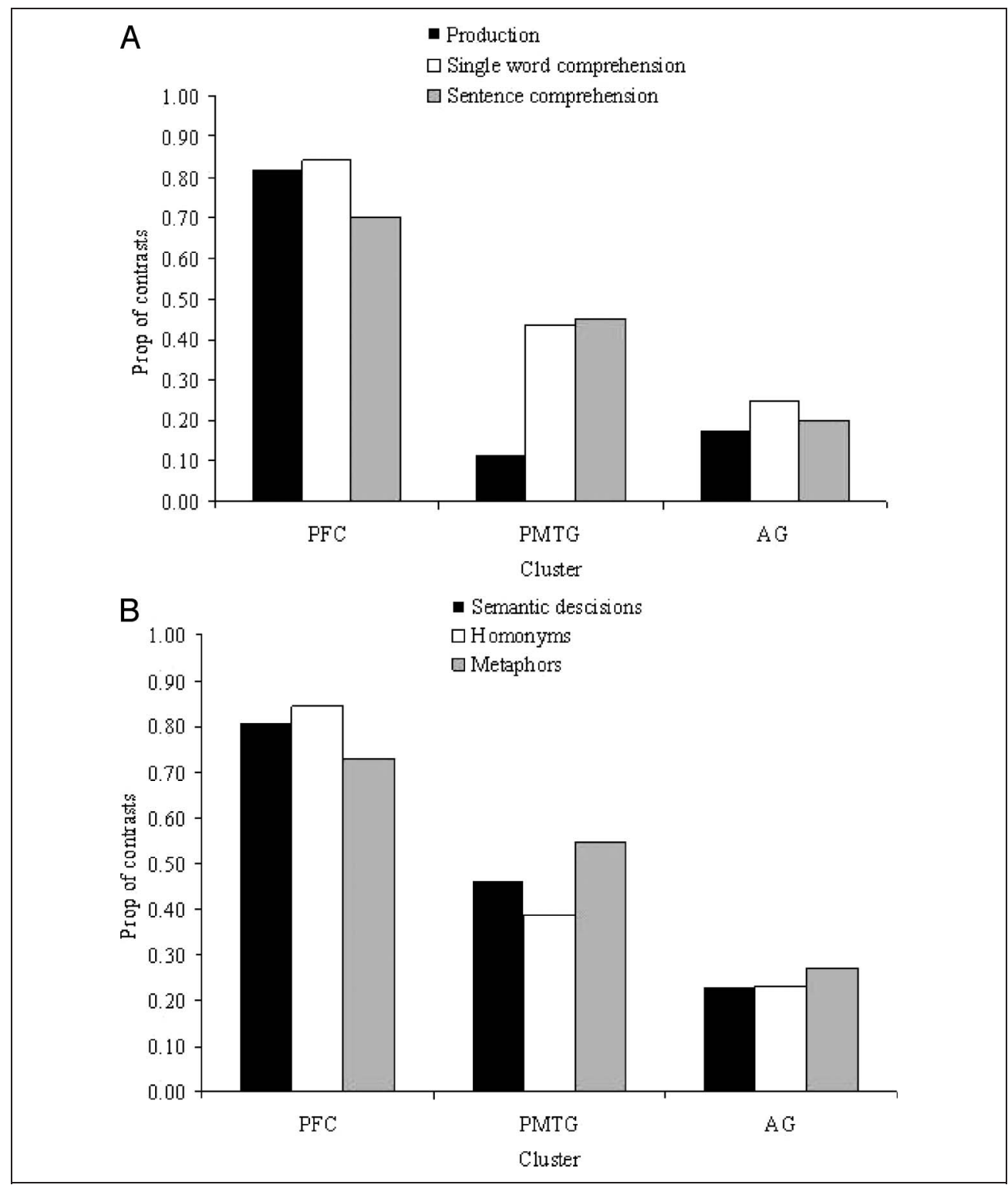

and receptive semantic tasks were equally likely to contribute peaks to the three anatomical clusters of interest (based on 53 studies: 14 production, 22 single word comprehension, 17 sentence comprehension). Figure $2 \mathrm{~A}$ reveals that regardless of the expressive/receptive dimensions of the task, studies were more likely to contribute to pFC cluster than either pMTG or AG $\left(\chi^{2}(2)=\right.$ 50.5, two-tailed $p<.001)$. Interestingly, expressive and receptive tasks (with either words or sentences) contributed equally to pFC $\left(\chi^{2}(2)=1.6, p>.4\right)$ and AG clusters $\left(\chi^{2}(2)<1\right)$. In contrast, production tasks contributed fewer peaks to the pMTG cluster relative to both receptive tasks with words $\left(\chi^{2}(1)=5.1, p=.02\right)$ and sentences $\left(\chi^{2}(1)=4.8, p=.03\right)$. Figure 3 presents separate ALE maps for semantic tasks requiring production and receptive comprehension (shown in blue-green). Most notably, receptive semantic tasks produced activation peaks that overlapped strongly with the original combined analyses (represented in red-yellow in Figure 3). Production tasks showed a similar pattern of activation in $\mathrm{pFC}$ and AG, but no evidence of peaks arising in pMTG, suggesting that the latter area is principally involved in receptive comprehension.

We next assessed the possibility that different types of semantic control may influence the likelihood of studies contributing peaks to each of the anatomical clusters of interest. Tasks were grouped according to whether they required (1) a comparison or other type of semantic decision (e.g., single items with relatively static meanings, had to be compared with each other-categorization, comparison, synonym judgment, etc.) or (2) processing of stimuli involving conflict because of intrinsic ambiguitythat is, homonyms and metaphors. The analyses were based on 38 studies (20 semantic decisions, 8 homonyms, 10 metaphors). Figure 2B highlights that, as before, all types of task were more likely to contribute to the 
Figure 3. ALE maps for anatomical clusters activated by (A) expressive and (B) receptive semantic tasks. Anatomical clusters from the combined high $>$ low semantic regulation analysis (the same as Figure 1) are presented in red-yellow for ease of comparison. Blue-green represents clusters that were activated by production tasks (A: 14 studies) and receptive comprehension tasks (B: 23 studies), respectively. Both tasks generate overlapping activation clusters across all regions with the exception of pMTG. Data corrected for multiple comparisons using an FDR threshold of $p<.05$. The anatomical clusters of interest are outlined with a dashed line (see text for details).

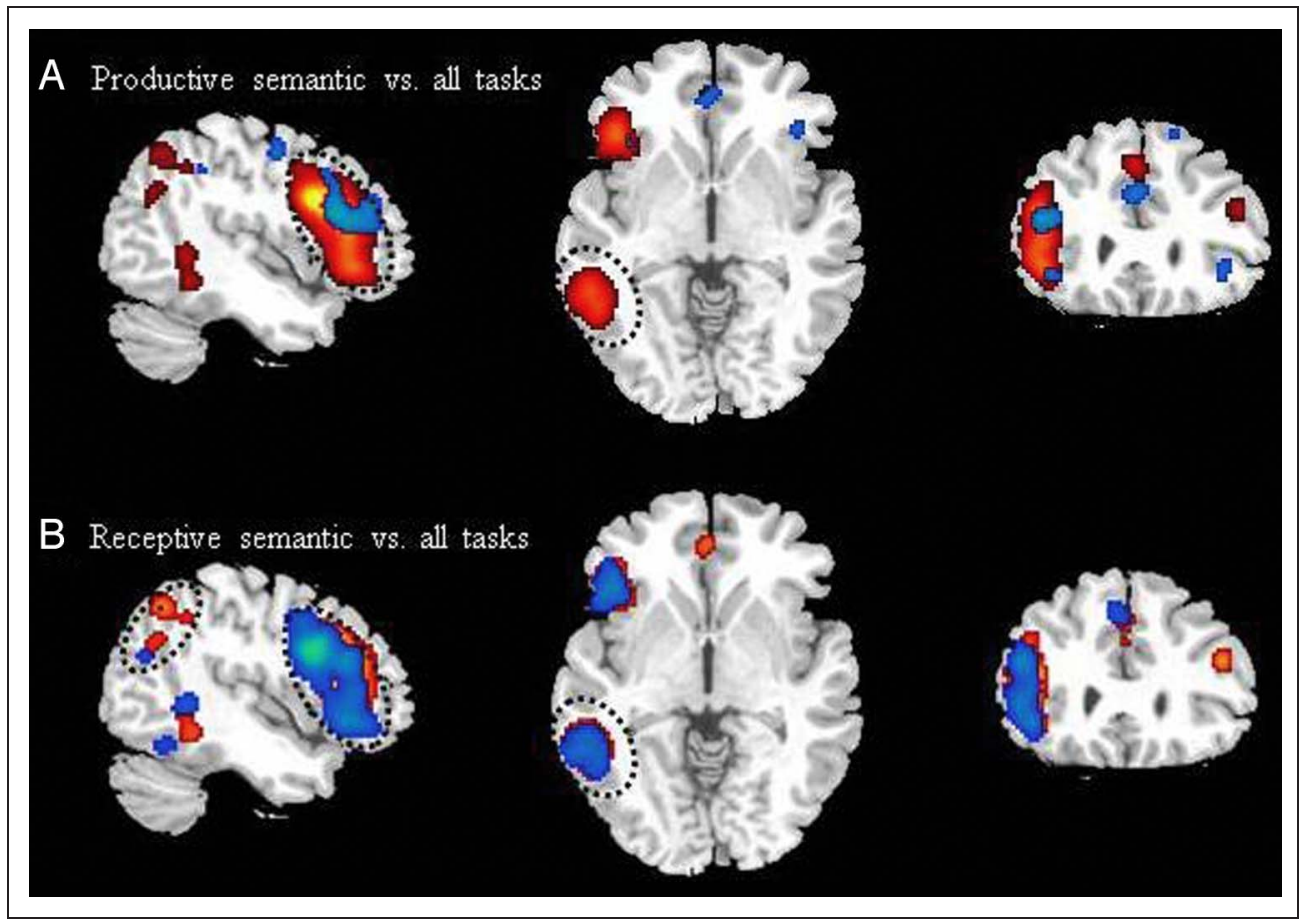

left pFC cluster than either pMTG or AG $\left(\chi^{2}(2)=31.8\right.$, two-tailed $p<.001)$. However, all types of executive manipulation contributed equally to the individual clusters $\left(\chi^{2}(2)<1\right)$. Figure 4 presents ALE maps for each of the three different types of executive semantic task. All three tasks show clear overlap with pFC, pMTG, and AG clusters from the first overall analysis (see Figure 1).

\section{Semantic Control versus Phonological Control}

As noted in the Introduction, SA patients have deficits in cognitive control that extend beyond the semantic do- main. This leads to the prediction that the regions that support semantic control may partially overlap with areas that underpin executive functions more generally. To investigate this hypothesis, we identified studies that directly compared executive semantic processing with other forms of executively demanding tasks: The vast majority of these studies (all bar three) compared semantic and phonological decisions (e.g., rhyme judgment, syllable decision), and we therefore focused our analysis on this specific subset of studies.

In the first analysis, we investigated the contrast between each type of executive control and its respective
Figure 4. Similarity of likelihood clusters across different executive semantic manipulations. Anatomical clusters from the combined high $>$ low semantic regulation analysis (the same as Figure 1) are presented in red-yellow for ease of comparison. Blue-green represents clusters that were activated by semantic decision tasks (A: 20 studies), tasks using homonyms (B: 8 studies), and studies using metaphors (C: 10 studies), respectively. Data corrected for multiple comparisons using an FDR threshold of $p<.05$. Anatomical clusters of interest are outlined with dashed lines.

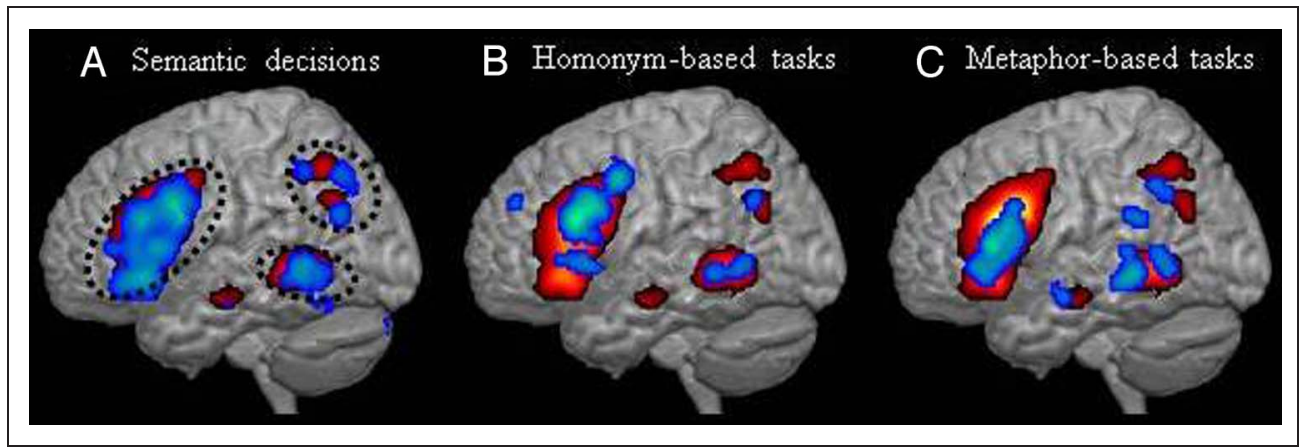


Figure 5. ALE maps for semantic control $>$ baseline and phonological control $>$ baseline. Analysis is based on eight studies that compared semantic tasks with baseline assessments and eight studies that compared phonological control with a low level condition. Red represents areas active for semantic tasks, blue represents areas active for phonological control, and pink conveys the areas that were active for semantic and phonological control. All regions except posterior temporal were involved in both types of control: (A) left hemisphere, (B) right hemisphere, and (C) medial left hemisphere. Data corrected for multiple comparisons using an FDR threshold of $p<.05$. Only clusters with an extent of $25 \mathrm{~mm}^{3}$ are displayed.

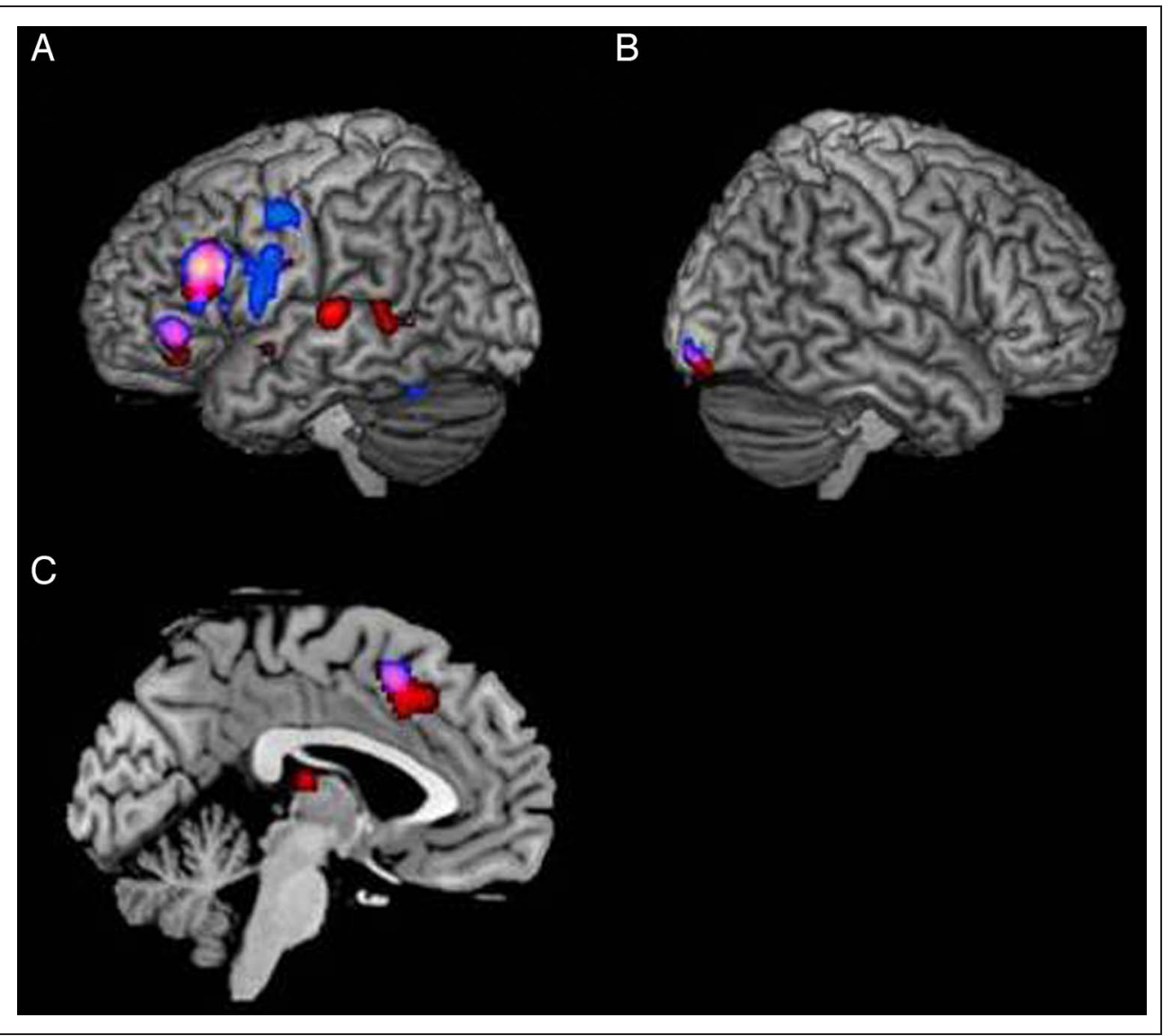

lower-level baseline (e.g., high semantic control > visual decisions or rest; high phonological control $>$ visual decisions or rest) to ascertain the degree of overlap between the networks involved in semantic and nonsemantic control. In the second set of analyses, we explored whether commonly activated areas exhibit graded specialization for semantic and nonsemantic tasks.

The studies that directly contrasted high-control semantic tasks against baseline tasks/rest revealed a network that overlapped with the previous high $>$ low semantic control analysis (Figure 5 or Supplementary axial-slice version: presented in red/yellow, Table 2). However, because the current analysis did not subtract out relatively automatic semantic processing, additional activation was seen in regions associated with semantic representation and not control processes, such as the anterior fusiform (Binney et al., 2010; Mion et al., 2010). Two clusters of high likelihood were detected in the left inferior frontal lobe. The larger of the two was located in BA 45 and extended more dorsally toward premotor cortex (BA 6). A smaller ventral cluster was detected in BA 47. Reliable peak clusters in the posterior temporal lobe were detected in pMTG (BA 21), the superior temporal gyrus (pSTG: BA 22), and ventral fusiform areas (BA 37). Two additional clusters were detected in the left medial frontal cortex (BA 32) and right inferior frontal gyrus (BA 44/BA 45). Both of these areas were also found in the first set of analyses reported above.

Phonological control contrasted with baseline tasks/ rest generated a similar pattern of activation in ventral and medial pFC (Figure 5: presented in blue/green, Table 2). Two clusters, one in BA 44/BA 45 and the other in ventral BA 47, overlapped with those found in the semantic $>$ baseline analysis. Similarly, the cluster detected in the left anterior cingulate (BA 32) was similar to that found in the semantic > baseline tasks/rest analysis. In contrast, phonological control did not produce significant activation in pMTG, suggesting a circumscribed role for this area in semantic processing only. High-control phonological tasks also yielded activation in dorsal premotor cortex, consistent with a role for this region in explicit phonological judgments and verbal working memory.

This analysis suggested that lateral pFC contributes to both semantic and phonological processing. Our next set of analyses explored relative specialization for semantic and phonological control. Figure 6 (see Supplementary axial-slice version) provides ALE maps for studies that reported the following contrasts (1) semantic $>$ phonological control (presented in red/ yellow) and/or (2) phonological > semantic control (presented in blue/green). Semantic control produced stronger activation in $\mathrm{BA} 47$, whereas phonological control 
activated more dorsal regions in BA 44/BA 6. Additional clusters were generated in BA 44/BA 45 for semantics and BA 45/BA 46 for phonological control. Within inferior parietal cortex, semantic processing produced greater activation in ventral AG (vAG; BA 39), whereas SMG (BA 40) was relatively more involved in phonological processing. However, this vAG site did not overlap with the dAG activation revealed by the contrast of semantic tasks with high and low control demands. This suggests that vAG is unlikely to have an executive semantic role. Additionally, phonological processing recruited a larger network of bilateral structures in both the inferior and superior parietal lobes (BA 40/7). Replicating the findings from the baseline comparisons, pMTG was only activated by executive semantic tasks (see Figure 6).

\section{Modality Specific versus Multimodal Control}

An important question, considering the multimodal nature of SA patients semantic deficits, is to establish whether left pFC, pMTG, and AG respond consistently across modality of presentation. This was impossible to assess satisfactorily in the current analysis because of an overwhelming bias in the literature: The majority of studies that manipulated semantic control used either visually presented words (51\%) or sentences (27\%). Far fewer studies used spoken words (9\%) and sentences (3\%), and only a small minority of contrasts used pictures (7\%). Interestingly, all of the studies that used pictures involved dual presentation with written words. There is a clear need, therefore, for future studies to

Table 2. Activation Clusters Derived from the GingerALE Analysis of Semantic Control > Baseline and Phonological Control > Baseline

\begin{tabular}{|c|c|c|c|c|c|c|c|c|}
\hline \multirow[b]{2}{*}{ Cluster No. } & \multirow[b]{2}{*}{ Brain Region } & \multirow[b]{2}{*}{ Hemisphere } & \multirow[b]{2}{*}{ Volume $\left(\mathrm{mm}^{3}\right)$} & \multicolumn{3}{|c|}{$\begin{array}{l}\text { Talairach } \\
\text { Coordinates }\end{array}$} & \multirow{2}{*}{$\begin{array}{c}\text { Brodmann's } \\
\text { Areas }\end{array}$} & \multirow{2}{*}{$\begin{array}{l}\text { Maximum } \\
\text { ALE Value }\end{array}$} \\
\hline & & & & $x$ & $y$ & $z$ & & \\
\hline \multicolumn{9}{|c|}{ Semantic > Baseline/Control Task } \\
\hline 1 & Ventral pFC & Left & 6360 & -42 & 22 & 20 & 45 & 0.03 \\
\hline 2 & Anterior cingulate & Left & 2344 & -2 & 18 & 45 & 32 & 0.01 \\
\hline 3 & Posterior inferior temporal cortex & Left & 1560 & -35 & -47 & -20 & 37,20 & 0.01 \\
\hline 4 & Ventral pFC & Left & 1552 & -40 & 36 & -6 & 47 & 0.01 \\
\hline 5 & MTG & Left & 1352 & -52 & -45 & 2 & 21,22 & 0.01 \\
\hline 6 & Superior temporal gyrus & Left & 1080 & -56 & -26 & 4 & 22 & 0.01 \\
\hline 7 & Visual cortex & Right & 1016 & 23 & -91 & -11 & 18 & 0.01 \\
\hline 8 & Premotor cortex & Left & 888 & -47 & -3 & 25 & 6 & 0.01 \\
\hline 9 & Anterior fusiform & Left & 832 & -32 & -4 & -32 & 36 & 0.01 \\
\hline 10 & Ventral pFC & Right & 592 & 30 & 21 & 5 & 47 & 0.01 \\
\hline 11 & Thalamus & Left & 544 & -6 & -19 & 13 & - & 0.01 \\
\hline 12 & Superior temporal gyrus & Left & 312 & -46 & 1 & -8 & 22,38 & 0.01 \\
\hline 13 & Visual cortex & Left & 256 & -12 & -69 & 10 & 17 & 0.01 \\
\hline 14 & Ventral pFC & Right & 104 & 52 & 27 & 25 & 44,45 & 0.01 \\
\hline \multicolumn{9}{|c|}{ Phonological Control > Baseline/Control Task } \\
\hline 1 & Ventral pFC & Left & 11896 & -42 & 15 & 19 & $44,45,6$ & 0.02 \\
\hline 2 & Posterior fusiform gyrus & Left & 1568 & -38 & -50 & -21 & 37 & 0.01 \\
\hline 3 & Premotor cortex & Left & 960 & -47 & -6 & 42 & 6 & 0.01 \\
\hline 4 & Visual cortex & Right & 864 & 23 & -91 & -7 & 18 & 0.01 \\
\hline 5 & Anterior cingulate & Left & 648 & -1 & 13 & 50 & 32 & 0.01 \\
\hline 6 & Ventral pFC & Left & 616 & -44 & 37 & -2 & 47,45 & 0.01 \\
\hline 7 & Anterior fusiform & Left & 432 & -30 & 0 & -34 & 36 & 0.01 \\
\hline 8 & Caudate nucleus & Left & 288 & -16 & 0 & 15 & - & 0.01 \\
\hline
\end{tabular}


Figure 6. ALE maps for direct comparisons of semantic and phonological control. Analysis is based on 15 studies that presented direct semantic > phonological executive contrasts and 14 studies that contrasted phonological > semantic control. Red represents areas that were relatively more active for semantic tasks, blue represents areas more active for phonological control, and pink conveys the areas that were active for semantic and phonological control: (A) left hemisphere, (B) right hemisphere, and (C) medial left hemisphere. Data corrected for multiple comparisons using an FDR threshold of $p<.05$ Only clusters with an extent of $100 \mathrm{~mm}^{3}$ are displayed.

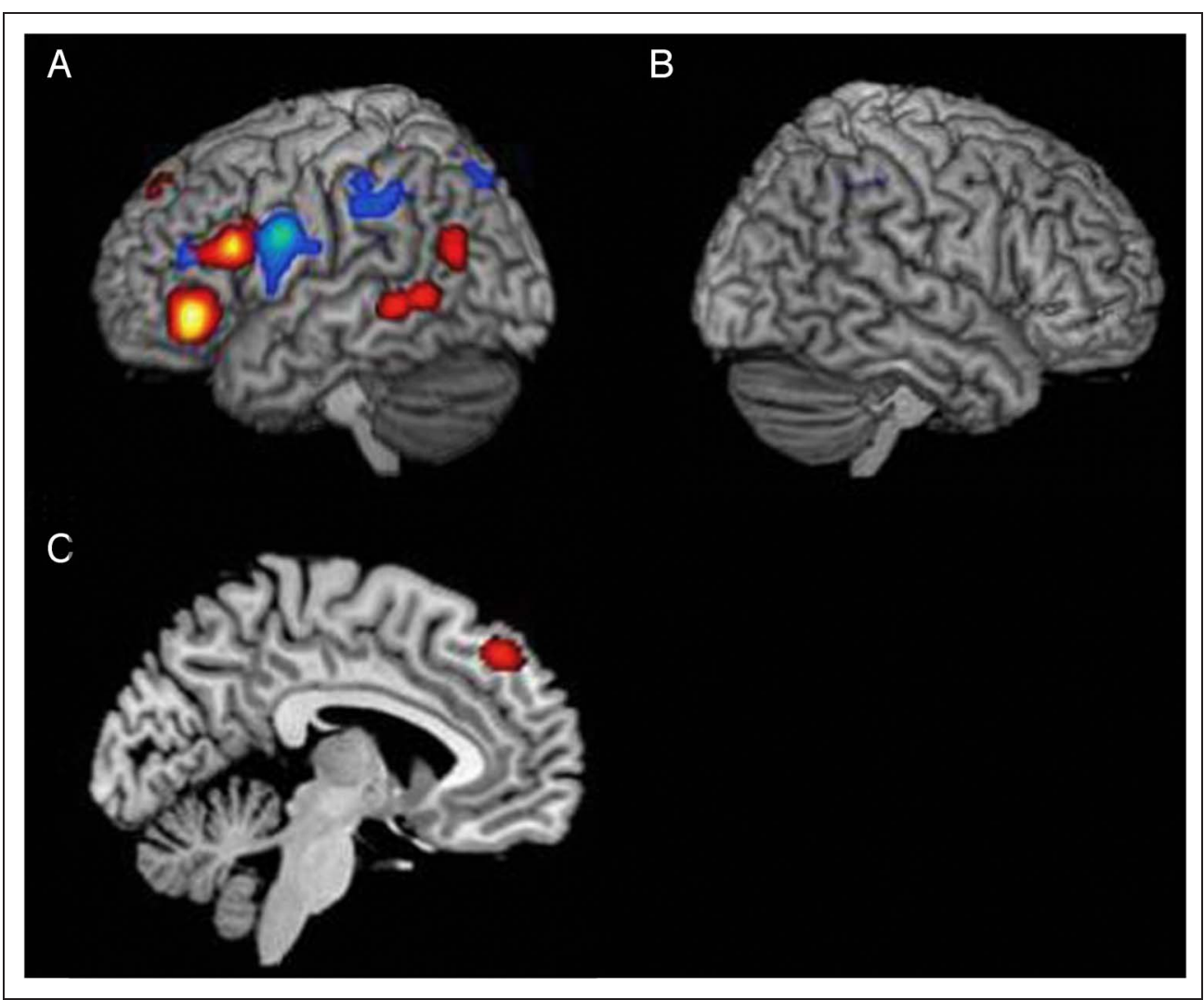

contrast semantic and cognitive control in the nonverbal domain.

\section{DISCUSSION}

Functional neuroimaging studies have made significant advances in clarifying the contribution and characteristics of the left ventral pFC to semantic control. In parallel, both contemporary and historical neuropsychological studies of SA reveal, in addition to ventral pFC, the importance of temporoparietal regions for the task-oriented regulation of conceptual knowledge. The central aim of the current meta-analysis was to test the degree of convergence between these two literatures using a theoretical framework that distinguishes between (1) conceptual representations underpinned by the ATL and linked modality-specific brain regions (in discrete portions of posterior temporal, frontal, and parietal cortex) and (2) semantic control processes, instantiated across yoked regions within $\mathrm{pFC}, \mathrm{dAG}$, and $\mathrm{pMTG}$, which regulate and shape semantic activation. A number of themes motivated by the neurocognitive profile of SA patients were used to explore the functional neuroimaging literature focusing on studies that have contrasted high and low semantic control demands. Each of these themes and their associated findings are summarized below:

(1) Semantic control is underpinned by a coupled system with distributed components extending beyond pFC.
Executive semantic processing modulated activation in a bilateral network of regions including left and right ventral and dorsal pFC, left posterior middle temporal cortex (pMTG), dorsal and anterior portions of AG, bordering IPS and SMG, and anterior cingulate regions. Several of these brain regions consistently activated by the executive control demands of semantic tasksleft pFC, pMTG, and dAG-overlapped with the most common areas of damage in SA patients who have impaired semantic control (Noonan et al., 2010; Jefferies \& Lambon Ralph, 2006; Head, 1926). Therefore, it is likely that left pFC, pMTG, and dAG work in concert to regulate semantic activation in a taskand context-sensitive fashion.

(2) Semantic control may be multifaceted with specific regions within the network supporting different aspects of executive semantic processing. Our analysis shows that left pFC and dorsal/anterior AG were consistently activated by all types of executive semantic manipulation (e.g., categorization, comparison, ambiguity processing), irrespective of the expressive/receptive nature of the task. This finding is consistent with SA patients' difficulties on a wide range of semantic tasks that place high demands on executive processes, including manipulations of the control demands of expressive tasks: Both $\mathrm{pFC}$ and temporoparietal patients show effects of cues and miscues on picture naming, for example (Noonan et al., 2010). In contrast, pMTG only contributed to receptive semantic tasks within 
our meta-analysis. This finding is again broadly consistent with neuropsychological and TMS evidence: Focal lesions and TMS stimulation of left ventral pFC both give rise to heightened competition from semantically related competitors during picture naming (Schnur et al., 2009). In sharp contrast to this strong dissociation between left pFC and pMTG in the expressive domain, TMS to LIFG and pMTG disrupts comprehension tasks with high control demands to an equal degree (Whitney et al., 2010).

(3) Semantic control shares some neurocognitive resources with domain-general control. We compared high-control tasks within the semantic and phonological domains, revealing overlapping activation in ventral and medial pFC. Similarly, other recent studies have revealed parallel responses to executive demands in pFC and IPS across a range of different domainsincluding semantic judgments (Barbey et al., 2012; Duncan, 2010; Spreng et al., 2010; Duncan, 2006; Duncan \& Owen, 2000). This is consistent with the association between semantic control and executive deficits seen in patients with SA. pFC and IPS show similar activation across a variety of attentional/ executive processes including set shifting, updating contextual information in working memory and inhibitory processing (Nee et al., 2007; Collette, Hogge, Salmon, \& Van der Linden, 2006), whereas lesions of these sites produce parallel deficits in visual attention (Peers et al., 2005). Moreover, TMS to dorsal pFC and IPS disrupts executive processes for both semantic and nonsemantic tasks (Whitney, Kirk, O'Sullivan, Lambon Ralph, \& Jefferies, 2012; Nagel et al., 2008), consistent with the finding that anterior and posterior lesions in SA produce comparable deficits of semantic and executive control (Noonan et al., 2010). Although we did not observe reliable activation of IPS for highcontrol semantic and phonological tasks in this metaanalysis, this null result is likely to reflect a lack of statistical power: Only a small subset of studies from the main analysis were suitable for inclusion in these contrasts (eight semantic and eight phonological studies).

There was also evidence of graded specialization of function within the distributed network underpinning semantic and nonsemantic control, which may be challenging to detect in studies of SA patients given their typically large lesions. Semantic tasks with high control demands elicited higher likelihood estimates in the most ventral parts of pFC (BA 47), whereas phonological tasks were associated more with activation in dorsal pFC and adjacent parts of premotor cortex (cf. Vigneau et al., 2006; Gough, Nobre, \& Devlin, 2005). pMTG was only activated by executively demanding semantic tasks and did not contribute to phonological control. Semantic tasks with high control demands also activated vAG, whereas phonological tasks yielded more activation of SMG. How- ever, because these contrasts compared semantic/ phonological control with low-level baseline tasks or rest periods, these activations are likely to reflect semantic and phonological processing in general and not only the control demands of the tasks. In addition, because the majority of neuroimaging studies in our meta-analysis manipulated control demands for linguistic stimuli, future research is needed to establish if each of these regions also respond to control demands within pictorial semantic tasks and to nonverbal aspects of executive control.

The regions highlighted in this study represent a subset of those found in previous large-scale meta-analyses, which focused on semantic cognition in general rather than a specific component of it (Visser et al., 2010; Binder et al., 2009). When compared, there is a clear emergent story that chimes directly with recent neuropsychological studies of different semantically impaired patient groups. Specifically, the full set of regions highlighted by these metaanalyses divide into two: (a) various anterior temporal and other regions for semantic representation (forming a hub-and-spoke framework: Pobric et al., 2010; Patterson, 2007) and (b) a network of frontal, dorsal/anterior AG and posterior middle temporal regions for semanticexecutive control. This same functional and anatomical division is found in the neuropsychological and rTMS literatures such that damage or stimulation to anterior temporal regions leads to impairment of semantic representations, whereas damage or stimulation to pFC, pMTG, or IPS/dAG compromises semantic control (Robson, Sage, \& Lambon Ralph, 2012; Pobric et al., 2010; Whitney et al., 2010; Lambon Ralph, Pobric, \& Jefferies, 2009; Schwartz et al., 2009; Pobric, Jefferies, \& Lambon Ralph, 2007; Jefferies \& Lambon Ralph, 2006).

Our meta-analysis is also consistent with studies of resting-state functional connectivity, which have revealed a "frontoparietal control network" comprising lateral (ventral and dorsal) pFC, dorsomedial pFC, dorsal and anterior parts of $\mathrm{AG}$, anterior cingulate, and a region of pMTG anterior to visual area $\mathrm{MT}^{+}$(Spreng et al., 2010; Vincent et al., 2008). All of these brain regions were revealed by our contrast of tasks with high as opposed to low semantic control demands. The "frontoparietal control network" lies between areas primarily involved in visuospatial attention (e.g., IPS/superior parietal lobule, $\mathrm{MT}^{+}$, posterior and medial $\mathrm{pFC}$ ) and brain regions associated with semantic processing and autobiographical memory, irrespective of control demands (i.e., anterior-to-mid temporal lobe regions plus ventral/posterior parts of AG; see Results and Discussion below). As such, it is thought to integrate and flexibly allocate attention to internal memories/ concepts and external inputs (Spreng et al., 2010; Vincent et al., 2008). Similarly, recent functional neuroimaging studies that analyze continuous visually presented films or spoken narratives using variable temporal receptive windows found that both posterior temporal-to-inferior 
parietal and prefrontal regions are implicated in the integration of meaningful stories over longer timescales (Lerner, Honey, Silbert, \& Hasson, 2011). In keeping with the patient and rTMS data, these studies revealed overlap in the inferior parietal region for spoken narratives and visually presented films, implicating this posterior region in multimodal on-line integration of context. Given these various neuroscience and neuropsychological results, the following discussion considers the potential role of each of the sites within the semantic control network, combining the outcomes of this meta-analysis with existing theories and previous research.

\section{Ventral pFC (BA 44, BA 45, BA 47)}

We replicated the well-established finding that left ventral pFC (BA 47, BA 45, BA 44) makes a clear and undisputed contribution to semantic control. The meta-analysis contrasting high-control semantic and phonological tasks also confirmed that, although this region contributes to a wide variety of tasks involving semantic control, including semantic decisions, homonym and metaphor judgments, and expressive semantic tasks, the most anterior parts of this site (pars orbitalis; BA 47) make a stronger contribution to semantic tasks whereas posterior regions (pars opercularis, BA 44, plus ventral premotor cortex) play a greater role in phonological tasks. Similar results have been reported previously in functional neuroimaging and TMS investigations (Sharp et al., 2010; Gough et al., 2005; Devlin et al., 2003; Gold \& Buckner, 2002) and in previous meta-analyses of language processing (Vigneau et al., 2006; Poldrack et al., 1999). This functional specialization is graded such that the whole of left ventral pFC, including BA 44/premotor cortex, responds to high versus low semantic control demands to some degree, whereas mid-ventral pFC (pars triangularis; BA 45) shows significant activation for both semantic and phonological tasks. Specialization of function within left ventral pFC could reflect differences in connectivity and/or processing domain across ventral pFC (Xiang, Fonteijn, Norris, \& Hagoort, 2010; Friederici, 2009; Saur et al., 2008) or, alternatively, the varying requirements that semantic and phonological tasks place on control processes. For example, the two-process model of lateral pFC (Badre \& D'Esposito, 2009) suggests that anterior pFC (BA 47) is important for high-level abstract control—such as determining the aspects of knowledge that might be relevant for a given judgment of semantic association. In contrast, more posterior $\mathrm{pFC}$ (BA 45/BA 44) is thought to be critical for selecting between competing alternatives and inhibiting task-irrelevant responses (Badre et al., 2005): control processes that semantic and phonological tasks/domains are likely to share.

Left posterior ventral pFC may also contribute, along with dorsolateral and medial prefrontal regions, to executive control beyond language. For example, Spreng et al. (2010) examined the overlap between the brain net- works recruited by planning in two domains: visuospatial planning (in the Tower of London task) and autobiographical planning (which draws strongly on semantic and episodic memory). Common areas of activation were identified in BA 45, as well as BA 9 and BA 6. However, our meta-analysis identified very few studies in the literature that have contrasted high and low executive demands across semantic and nonlinguistic tasks; almost all of the available comparisons were between semantics and phonology.

Whereas neurocognitive theories of semantic control largely focus on left ventral $\mathrm{pFC}$ - the site which produced the strongest concentration of activation foci in our metaanalysis - reliable activation was also detected in right ventral $\mathrm{pFC}$, indicating that semantic control emerges from a bilateral system. Numerous individual functional neuroimaging studies have reported a bilateral ventral $\mathrm{pFC}$ response to manipulations of the executive demands of semantic tasks, including many "classic" investigations of semantic control (e.g., Synder, Banich, \& Munakata, 2011; Badre et al., 2005; Wagner et al., 2001; ThompsonSchill et al., 1997). In our meta-analysis, this right-sided activation was reliable across different anatomical regions, including BA 44, BA 45, and BA 47. Because domaingeneral executive functions are also thought to emerge from a bilateral network including left and right pFC and IPS (Duncan, 2010), further research is required to establish the extent to which right ventral $\mathrm{pFC}$ shows graded specialization similar to the organization of function uncovered for left ventral pFC.

\section{Dorsal Medial pFC (BA 8 and BA 9)}

As noted by Binder et al. (2009), dorsal medial pFC, anterior to SMA (BA 6), is consistently activated by semantic tasks, although its contribution to conceptual processing is unclear and often overlooked. Binder et al. speculated that it might be involved in "self-guided" or "goal-directed" semantic retrieval, which is typically critical for control tasks with high control demands. In this meta-analysis, it was activated by the contrast of high $>$ low semantic control and also showed activation for executively demanding phonological tasks, indicating that its contribution is not domain-specific. In line with these findings, dorsal medial $\mathrm{pFC}$ is thought to be a key component of the "frontoparietal" control network (Duncan, 2010; Vincent et al., 2008; Duncan \& Owen, 2000).

\section{AG (BA 39)}

In the meta-analysis of neuroimaging studies conducted by Binder et al. (2009), left AG was the region most consistently activated by semantic tasks but its precise role in semantic cognition remains elusive. This might partly reflect the fact that AG is a large cortical region, associated with diverse functions (for reviews, see Cabeza, Ciaramelli, 
\& Moscovitch, 2012a; Seghier, Fagan, \& Price, 2010). Critically, AG is thought to comprise several functionally dissociable areas (Seghier et al., 2010; Uddin et al., 2010; Caspers et al., 2008; Vincent et al., 2008), and these could contribute differentially to semantic representation and control. Resting-state functional connectivity studies suggest that dorsal anterior AG (bordering IPS and SMG) forms part of the "frontoparietal" control network (Spreng et al., 2010; Vincent et al., 2008)—-the integrity of which is likely to be critical to the ability to focus semantic processing on task-/context-relevant aspects of meaning, as well as executive control within other domains. In line with this hypothesis, Xiang et al. (2010) found strong connectivity between BA 45 and the dorsal and anterior parts of IPL, close to our dAG/IPS site. In contrast, BA 47 showed stronger connectivity to mid-AG (Xiang et al., 2010). An even more vAG site (bordering posterior temporal cortex) shows activity correlated with ATL (linked to semantic representation) and limbic areas (posterior cingulate and medial OFC) and relatively little connectivity with any part of LIFG (Vincent et al., 2008).

Comparisons of functional activation during semantic tasks and resting-state scans also point to separable AG regions (Seghier et al., 2010). Some AG regions show activations to semantic and nonsemantic stimuli, whereas others are part of the "default-mode network" (i.e., are active during rest) and show stimulus-driven deactivations. Seghier et al. (2010) identified three AG regions with distinct response profiles (dAG at approximately $z=+40$, mid-AG at $z=+30$ and $\mathrm{vAG}$ at $z=+20$; in MNI space). Reliable activations were detected within all of these regions in the current meta-analysis, and we have adopted the labels $\mathrm{dAG} / \mathrm{IPS}$, mid-AG, and vAG throughout this study. Importantly, activations within these regions were revealed by different contrasts (see labels in Tables 1 and 3), consistent with the view that they have somewhat different functional roles.

\section{Left dAG}

The most consistently activated site within inferior parietal cortex, revealed by our comparison of semantic tasks with high and low control demands, was situated at the boundary of dorsal/anterior AG, SMG, and IPS (see Table 1). We refer to this location as "dAG/IPS," while acknowledging that some of the peaks contributing to activation within

Table 3. Activation Cluster Derived from the GingerALE Analysis of Semantic versus Phonological Control Studies

\begin{tabular}{|c|c|c|c|c|c|c|c|c|}
\hline \multirow[b]{2}{*}{ Cluster No. } & \multirow[b]{2}{*}{ Brain Region } & \multirow[b]{2}{*}{ Hemisphere } & \multirow[b]{2}{*}{ Volume $\left(\mathrm{mm}^{3}\right)$} & \multicolumn{3}{|c|}{$\begin{array}{l}\text { Talairach } \\
\text { Coordinates }\end{array}$} & \multirow[b]{2}{*}{ Brodmann's Areas } & \multirow{2}{*}{$\begin{array}{l}\text { Maximum } \\
\text { ALE Value }\end{array}$} \\
\hline & & & & $x$ & $y$ & $z$ & & \\
\hline \multicolumn{9}{|c|}{ Semantic > Phonological Control } \\
\hline 1 & Ventral pFC & Left & 4168 & -42 & 34 & -6 & 47,45 & 0.02 \\
\hline 2 & Ventral pFC & Left & 3928 & -47 & 18 & 22 & 45,44 & 0.02 \\
\hline 3 & pMTG & Left & 2144 & -54 & -49 & -1 & 21,37 & 0.01 \\
\hline 4 & AG (ventral) & Left & 1984 & -44 & -66 & 21 & 39,37 & 0.01 \\
\hline 5 & Dorsolateral pFC & Left & 1096 & -6 & 43 & 44 & 9,8 & 0.01 \\
\hline 6 & Inferior temporal cortex & Left & 1072 & -36 & -22 & -21 & 20 & 0.01 \\
\hline 7 & Premotor cortex & Left & 680 & -34 & 4 & 50 & 6 & 0.01 \\
\hline 8 & Anterior cingulate & Left & 480 & -12 & 43 & 1 & 32,11 & 0.01 \\
\hline \multicolumn{9}{|c|}{ Phonological > Semantic Control } \\
\hline 1 & Ventral pFC & Left & 4304 & -52 & 0 & 23 & 44,6 & 0.02 \\
\hline 2 & SMG & Left & 3984 & -42 & -42 & 37 & 40 & 0.02 \\
\hline 3 & Superior parietal lobule & Left & 2624 & -21 & -67 & 45 & 7 & 0.01 \\
\hline 4 & Superior parietal lobule & Right & 1424 & 26 & -62 & 34 & 7,19 & 0.01 \\
\hline 5 & $\mathrm{SMG}$ & Right & 1232 & 40 & -43 & 41 & 40 & 0.01 \\
\hline 6 & Ventral pFC & Left & 1032 & -38 & 32 & 18 & 45 & 0.01 \\
\hline 7 & Premotor cortex & Left & 432 & -7 & -3 & 59 & 6 & 0.01 \\
\hline 8 & SMG & Right & 104 & 43 & -51 & 49 & 40 & 0.01 \\
\hline
\end{tabular}


this site lie within SMG (see Appendix Table 2A). Comparison with the cytoarchitectural map of Caspers et al. (2008) confirms that the peak of this activation lies, with approximately equal probability, within dAG ( $\mathrm{PGa})$, posterior SMG (PFm), and IPS (hIP1).

dAG, bordering IPS/SMG, shows activation in response to both word and picture semantic tasks and also to nonsemantic stimuli (relative to fixation; Seghier et al., 2010), showing it is not within the "default network"; instead, this region is thought to contribute to a bilateral frontoparietal control network, which underpins domain-general executive processing (Duncan, 2010; Spreng et al., 2010; Vincent et al., 2008). A similar site in the right hemisphere was also identified by the contrast between high and low semantic control demands, as predicted by this hypothesis (see Table 1). Our peak overlaps with shared activation for visuospatial and autobiographical planning tasks identified by Spreng et al. (2010) - in contrast, unique activation for visuospatial planning occurred in an arc encompassing superior parietal cortex and $\mathrm{MT}^{+}$, whereas activity specific to autobiographical planning was focused on the "default network" (anterior-to-mid temporal areas, medial pFC, posterior cingulate, and mid-AG). This site may therefore allocate attention to the external world or internal memory representations, depending on task demands, and integrate these sources of information about the world, in a goal-driven fashion (Spreng et al., 2010; Vincent et al., 2008). This proposal is consistent with the suggestion by Binder et al. (2009) that AG underpins novel conceptual combination, because this process also involves selective attention to aspects of conceptual knowledge and integration of these elements to create a new concept.

Similar functional claims have been made for the region of IPS bordering our dAG/IPS site: indeed, the resting-state functional connectivity analysis of Vincent et al. (2008) revealed that $\mathrm{dAG}$ and neighboring IPS have similar patterns of functional connectivity. TMS to left IPS has been shown to disrupt executively demanding tasks within both the semantic and non-semantic domains (Whitney et al., 2012; Nagel et al., 2008). Moreover, our activation cluster in dAG/IPS is similar to the peak response observed in IPS when participants orient their attention to particular semantic features or categories, as well as to particular spatial locations following a cue (Cristescu, Devlin, \& Nobre, 2006). Therefore, left dAG/IPS may contribute to semantic control by allocating attention toward relevant aspects of knowledge for a given task or context, that is, through adaptive coding of task-critical information (Woolgar, Hampshire, Thompson, \& Duncan, 2011) in much the same way as it directs spatial attention to taskrelevant locations. Support for this hypothesis was provided by a recent TMS study in which stimulation of left IPS produced specific disruption of semantic decisions, which required a selective focus on a single feature (e.g., color-match "stop sign" with "tomato"). There was no effect on challenging semantic decisions based on weak global semantic associations, perhaps because these did not require the task-driven allocation of attention to specific semantic features. In contrast, stimulation of left ventral pFC and pMTG disrupted both types of semantic tasks with high-control demands, relative to a low-control condition (Whitney et al., 2012).

\section{Left Mid-AG}

The functioning of this area in semantic cognition and other domains is a puzzle and requires future studies to explore the nature of the associated cognitive processes in more detail. The high $>$ low executive semantic contrast used in this meta-analysis produced a small concentration of peaks within mid-AG. Comparison with the cytoarchitectural map of Caspers et al. (2008) confirmed that the peak of this activation fell within AG (with somewhat higher probability for PGp than PGa). At face value, this would suggest that, like the dAG-IPS, the MAG is also a part of a frontoparietal network for executive processing that is engaged by demanding semantic tasks. This conclusion does not seem to fit, however, with a range of other findings and meta-analyses of the neuroimaging literature. First, mAG has been associated with the semantic "richness" of stimuli even where task demands are matched. For example, it shows relatively greater activation (or rather less deactivation-see below) for semantic decisions as opposed to phonological decisions matched in terms of sensory and executive demands (Binder et al., 1999, 2009). Additionally, this site shows a relatively stronger response (less deactivation) to concrete than abstract concepts, although these items are easier to process (Wang, Conder, Blitzer, \& Shinkareva, 2010; Binder, Westbury, McKiernan, Possing, \& Medler, 2005). These findings seem to point toward a role of the MAG in semantic representation, particularly for "rich" multimodal concepts (Binder et al., 2009). Indeed, the fact that mAG is least active for the most "difficult" items (i.e., those with long decision/processing times such as abstract $>$ concrete words, nonwords $>$ words, etc.) appears to be inconsistent with the finding from this meta-analysis that $\mathrm{mAG}$ is associated with the more executively demanding semantic tasks.

The mAG conundrum is made even more complex in two further respects. First, it is part of set of regions that are associated with the "default mode network" (i.e., it shows activity in the absence of an active task; Raichle et al., 2001). Indeed, mAG was identified by Seghier et al. (2010) as the focus of overlap between a significant concreteness effect (concrete $>$ abstract) and the default network. This means that the area deactivates to concrete items yet even more so for abstract items. Additional studies are required to search for an explanation for why this region deactivates differentially depending on the type of stimulus yet other parts of the semantic network exhibit purely positive activations. The second complexity relates to the fact that this same region has been implicated in a very wide variety of cognitive domains that 
include semantic processing but also extend to episodic and autobiographical recall, syntax, number processing, theory-of-mind tasks, and "bottom-up" attention (Cabeza et al., 2012a; Cabeza, Ciaramelli, \& Moscovitch, 2012b). Future studies are needed to compare across these processing domains carefully and simultaneously-not only in terms of identifying a computational process that they all share but also to map exactly which parts of the ventral IPL are associated with each processing domain (Hutchinson et al., 2012; Nelson, McDermott, \& Petersen, 2012).

\section{vAG}

We observed reliable activation likelihood estimates within vAG when control-demanding semantic tasks were contrasted with phonological tasks. Because (i) this analysis did not specifically focus on control demands (i.e., both phonological and semantic tasks required some degree of control) and (ii) vAG was not highlighted in the main likelihood estimate when high-control semantic tasks were contrasted with low-control tasks, we suggest that this site plays a key role in semantic processing irrespective of control demands.

This view is consistent with findings from several other groups. As noted above, mid-AG to vAG is strongly connected to anterior-to-mid temporal lobe areas (Spreng et al., 2010; Vincent et al., 2008). Spitsyna, Warren, Scott, Turkheimer, and Wise (2006) found that temporaloccipital-parietal cortex, overlapping with our vAG site, was commonly activated by naturalistic comprehension tasks involving speech and written words, along with two sites in the ATL (lateral temporal pole and anterior fusiform gyrus). These passive listening/reading tasks were designed to minimize meta-cognitive demands and did not involve explicit semantic judgments although they would require on-line buffering and integration of information over time. Likewise, Seghier et al. (2010) demonstrated that vAG shows activation for meaningful items but deactivation for meaningless items - this site therefore shows a different response profile to the "default network," which has been argued to contribute to goal-driven cognitive activity (Spreng et al., 2010).

\section{pMTG}

The temporal lobe is often considered to be a repository of semantic representations (e.g., Binder et al., 2009): therefore, our finding - that the second strongest cluster in the comparison of high- and low-control semantic tasks (after left ventral pFC) fell within left pMTG-is novel and striking. pMTG lies anterior to motion perception area V5/MT and responds strongly to tool use and actions (Kable, Kan, Wilson, Thompson-Schill, \& Chatterjee, 2005; Chao, Haxby, \& Martin, 1999), suggesting a role for this site in representing movements and actions asso- ciated with objects (i.e., pMTG might form one of the "spokes" within the hub and spokes model). Nevertheless, in comprehension tasks (including those not tapping tool/ action knowledge), the response in pMTG appears tightly coupled whilst pMTG and ventral pFC are known to have strong anatomical and functional connections (Turken \& Dronkers, 2011; Xiang et al., 2010; Saur et al., 2008; Catani, Jones, \& Ffytche, 2005). In resting-state functional connectivity studies, a region of pMTG overlapping with the activation shown in Figure 1/Table 1 correlated with both (i) the regions in the frontoparietal system (e.g., pFC; IPS/ $\mathrm{dAG}$ ) and (ii) the temporal lobe memory system (e.g., ATL; Spreng et al., 2010; Vincent et al., 2008). Moreover, TMS to ventral pFC and pMTG produced equal disruption of two tasks with high semantic control demands tapping the controlled retrieval of distant associations and the selection of specific semantic features respectively but left a low-control semantic task unaffected (Whitney et al., 2010). This suggests that ventral pFC and pMTG are essential components of a distributed cortical system underpinning executive semantic processing.

Several researchers have already noted that the response in pMTG within individual fMRI studies is not consistent with the notion of a passive store of semantic attributes and have argued instead that pMTG may be involved in the strategic retrieval of semantic information (Gennari et al., 2007; Gold et al., 2006). We suggest that pMTG may be crucial for the flexible processing of concepts, such that semantic activation is focused on aspects of meaning that are appropriate to the task or context. This proposal is compatible with Turken and Dronkers's (2011) suggestion that interactions between ventral pFC and pMTG allow selected aspects of meaning to be sustained in STM such that they can be integrated into the overall context of the sentence (or task). This component of semantic control could conceivably overlap with action representation in pMTG, because actions must also be flexibly controlled to suit the context or task-for example, we can retrieve very different actions for "shoe" if the task is to bang in tent pegs rather than fasten our laces.

Although the role of pMTG in language comprehension is emphasized in some theoretical accounts-for example, acting as an interface between speech representations within superior temporal gyrus and distributed conceptual representations (Hickok \& Poeppel, 2004, 2007)—studies reveal a multimodal response: pMTG shows overlapping activation in response to semantic tasks involving either pictures or words (Visser, Jefferies, Embleton, \& Lambon Ralph, in press; Vandenberghe, Price, Wise, Josephs, \& Frackowiak, 1996) and TMS to this region disrupts semantic judgments presented in both modalities equally (Hoffman, Pobric, Drakesmith, \& Lambon Ralph, 2011). Likewise, contrasts of aphasic patients with verbal only versus multimodal semantic impairments indicate that verbal-only deficits are associated with pSTG/TPJ damage whereas multimodal deficits reflect damage to pMTG and AG regions (Robson et al., 2012; Chertkow et al., 1997). 
The suggestion that pMTG might play a critical role in capturing context-sensitive meaning is consistent with its multimodal response since the context-driven meanings of words and objects can be defined according to both linguistic and environmental/visual contexts; this proposal is also consistent with the notion that pMTG has particularly rich connections with other temporal, frontal, parietal, and occipital regions, allowing it to act as a cortical "hub" (Turken \& Dronkers, 2011).

Despite this strong connectivity, the current metaanalysis revealed some differences in the responses of pMTG and ventral pFC: (i) Although ventral pFC responded to the control demands of semantic tasks involving both production and comprehension, the contribution of pMTG was restricted to executive control of receptive tasks. (ii) pMTG also diverged from ventral pFC in that it was selectively involved in semantic processing-it was not implicated in executive control over other domains. These differences could potentially be explicable within the framework described above. Selection/inhibition (within ventral pFC) are domain-free executive processes that contribute to many different tasks and aspects of cognition. They are highly relevant in semantic production tasks such as picture naming, because a particular response must be retrieved and selected at a specific moment, whereas plausible alternative names and semantically-related competitors are avoided (e.g., saying "animal"/"springer spaniel" or "cat"/"bone" for "dog"). Comprehension tasks, such as matching words by semantic association, also require selection/inhibition; moreover, these aspects are often manipulated in studies of semantic control: for example, participants may be required to select a target in the face of strong distracters, yielding activation of ventral $\mathrm{pFC}$. Although production and comprehension tasks share this aspect of control, they perhaps differ in the importance of context (and therefore in the contribution of pMTG). The production tasks that contributed to this analysis involved (i) fluency (e.g., switching vs. semantically grouped word production), (ii) picture naming with and without semantic competitors, (iii) verb generation (from nouns with and without a single dominant response), and (iv) sentence completion (contrasting easy completions with the suppression of prepotent responses). These tasks focus on semantic retrieval under demanding conditions requiring inhibition/selection-but the nature of the words/concepts that are produced are not tuned to suit the context. In the first three tasks, the semantic context giving rise to production is relatively simple (i.e., a single word or picture) and trials vary in the ease with which a response can be identified and selected. In sentence completion, there is a richer context, but crucially this context does not change across high/low control conditions - instead the task is to produce a word that is consistent or inconsistent with the context, generating equivalent pMTG activation in both conditions.

In contrast, in comprehension tasks, it is necessary to work out which of several possible targets is the best fit to a probe word, given the task context provided by all of the words - that is, participants must construct a semantic context that provides a link between two words that do not have a strong and automatically retrieved association. Because this context is semantic in nature, we would not expect pMTG to be engaged by nonsemantic tasks requiring control (see also Lerner et al., 2011). Moreover, as noted by Vincent et al. (2008), pMTG shows correlated activity (at rest) with both the frontoparietal control network and anterior temporal areas. This connectivity might explain why pMTG makes a contribution to cognitive control, which is largely specific to the semantic domain.

Our meta-analysis made no attempt to separate the representational and control aspects of semantic cognition: Instead our focus was on revealing structures that reliably respond when control demands are elevated. In this context, we should consider a possible confound: tasks involving high semantic control demands conceivably involve the activation of a greater number of concepts or the maintenance of conceptual activity for a longer period. Indeed, because all semantic tasks necessarily involve both representation and control (at least to some degree), this confound is pervasive in the literature: activity within LIFG could be reinterpreted along these lines, in the absence of other constraining information. Moreover, although we have argued that LIFG and IPS/ $\mathrm{dAG}$ do not show the response characteristics of representational regions-because these regions can couple with either the "dorsal attention system" or the "resting state network" (Spreng et al., 2010), pMTG plays a more selective role in comprehension tasks and we have proposed that it contributes to context-sensitive aspects of meaning - a function that essentially bridges representation and control.

Because activity within the entire brain network supporting semantic cognition is expected to show modulation by both representational and control demands (but with sensitivity to each factor reflecting the specific role of each discrete region), stronger conclusions can be drawn from the small number of studies that have simultaneously manipulated control and representational demands within the same participants. These studies support our hypothesis that pMTG plays an important role in tasks with high semantic control demands, whereas more anterior temporal areas respond to representational demands. Using a double-prime paradigm, Whitney, Jefferies, and Kircher (2010a) asked participants to decide if the meaning of either of two prime words was related to the target. Left mid-inferior temporal gyrus (BA 20) was more strongly engaged when both interpretations of an ambiguous word were relevant to the task (e.g., "river" and "money" followed by BANK), because this condition maximized concept retrieval. In contrast, left pMTG and IFG showed greater activation when participants were asked to selectively retrieve the less frequent meaning of an ambiguous word (e.g., "river" and an unrelated word "clock"; followed by BANK), requiring inhibition of the dominant meaning. 


\section{Comparisons with Other Meta-analyses of Functional Neuroimaging Studies of Semantic Processing}

The current study examined the brain regions that play a greater role in semantic tasks when executive processes, such as selection and inhibition, are increased (i.e., contrasts presented in Table 1 and Figure 1). This work is therefore highly complementary to Binder et al.'s (2009) meta-analysis, which attempted to minimize the effect of executive demands within semantic cognition. In line with this difference, the meta-analysis of Binder et al. revealed considerably more activation in anterior to mid-temporal lobe regions, confirming the role of this area in semantic representation and processing irrespective of control demands. Other regions highlighted by Binder et al.'s (2009) analysis but not the current study include ventromedial pFC (including rostral cingulate gyrus) — which Binder et al. (2009) linked to the emotional aspects of meaning, and posterior cingulate-which they associated with episodic encoding. These processes/regions are presumably engaged by both high- and low-control semantic tasks and thus were not observed in the current meta-analysis. Perhaps surprisingly, both meta-analyses highlighted the importance of LIFG and dorsomedial pFC in semantic cognition. Binder et al. suggested that their LIFG activation might have arisen through imperfect control over the executive demands of the semantic and nonsemantic tasks they contrasted. Moreover, semantic paradigms may typically place strong demands on mechanisms that allocate attention to internal representations or that allow stimuli in the external world to be integrated with stored meanings, whereas the nonsemantic tasks used for comparison (which often involve phonological or visual decisions) might not involve these processes to the same degree (even when RT is matched). Similar task differences could potentially explain why both meta-analyses observed activation in dAG/IPS and mid-AG. Binder et al. associated their AG activation with semantic integration, which is often crucial in high-control tasks - and this function appears to be related to the contribution of frontoparietal control system (Vincent et al., 2008). Finally, in the current study, the contrast of high over low semantic control did not yield activation in more vAG regions (unlike the meta-analysis of Binder et al., which attempted to eliminate a contribution of general control). In contrast, the comparison of semantic and phonological tasks did show activation in this region-confirming that there are functional subdivisions within AG, with more dorsal regions contributing to control, and more ventral regions supporting semantic processing irrespective of control demands.

Unfortunately, given the nature of the current neuroimaging literature (which is populated mainly by studies that contrast semantic and phonological decisions), it was not possible to explore the response of ventral pFC, dAG, and pMTG to nonverbal aspects of executive semantic processing. Possibly for practical reasons, only a handful of studies have investigated semantic control using pictures or other nonverbal stimuli (e.g., environmental sounds). However, based on explorations of nonverbal processing in SA patients (e.g., Corbett, Jefferies, Ehsan, et al., 2009; Corbett, Jefferies, et al., 2009; Jefferies \& Lambon Ralph, 2006), our prediction would be that the semantic control network underpinned by ventral pFC, pMTG, and dAG would show greater activation as the executive requirements of nonverbal semantic tasks increased.

In conclusion, we propose that executive control over semantic processing is underpinned by a distributed neural network including bilateral pFC, left dAG, and left pMTG. We propose that this distributed interactive network is flexible, such that when the contribution of one region is disrupted, compensatory activation is observed in other parts of the network. Nevertheless, our metaanalysis suggests that these sites may have partially specialized roles: Although pFC and dAG/IPS contribute to the control of (i) production, (ii) comprehension, and (iii) nonsemantic processing, pMTG shows a more restricted response (control of comprehension only). We have interpreted this pattern within a theoretical framework in which pFC is involved in establishing current goals for semantic cognition (i.e., top-down control) plus selection/inhibition, left dAG contributes to the orientation of attention to task-relevant concepts and left pMTG forms a multimodal representation of context, which captures the relationship between task-relevant aspects of meaning in a flexible way. 
APPENDIX 1. Characteristics of the Neuroimaging Studies Contributing to the Contrast of High $>$ Low Semantic Control

\begin{tabular}{|c|c|c|c|c|c|c|}
\hline Authors & Imaging Type & Analysis Category & Task & Contrast & Modality & Coverage \\
\hline Allen et al. (2008) & fMRI & Verbal production & Hayling sentence completion task & Nonsalient $>$ Salient completion & Written sentences & Whole brain \\
\hline Amunts et al. (2004) & fMRI & Verbal production & Verbal fluency & Category $>$ Rote (days of the week, months) & Spoken words & Whole brain \\
\hline Assaf et al. (2006) & fMRI & Semantic decision & Object recall from feature pairs & Target present $>$ Target absent trials & Written words & Whole brain \\
\hline Assaf et al. (2006) & fMRI & Semantic decision & Associative decisions task & Associated $>$ Nonassociated trials & Written words & Whole brain \\
\hline Badre et al. (2005) & fMRI & Semantic decision & Semantic relatedness judgment & Weak $>$ Strong probe-target association & Written words & Whole brain \\
\hline Badre et al. (2005) & fMRI & Semantic decision & Semantic relatedness judgment & Four $>$ Two word choices & Written words & Whole brain \\
\hline $\begin{array}{l}\text { Bedny, McGill, \& } \\
\text { Thompson-Schill (2008) }\end{array}$ & fMRI & Homonyms & Semantic relatedness judgment & Ambiguous > Unambiguous words & Written words & ROI \\
\hline Bedny et al. (2008) & fMRI & Homonyms & Semantic relatedness judgment & Ambiguity Conjunction & Written words & Whole brain \\
\hline Bottini et al. (1994) & PET & Metaphors/idioms & Sentence plausibility judgment & Metaphorical > Literal meaning & Written sentences & Whole brain \\
\hline $\begin{array}{l}\text { Boulenger, Hauk, \& } \\
\text { Pulvermuller (2009) }\end{array}$ & fMRI & Metaphors/idioms & Sentence comprehension & Idiomatic $>$ Literal meaning & Written sentences & ROI \\
\hline $\begin{array}{l}\text { Bunge, Wendelken, Badre, } \\
\text { \& Wagner (2005) }\end{array}$ & fMRI & Semantic decision & Semantic relatedness judgment & Weak $>$ Strong probe-target association & Written words & ROI \\
\hline Chan et al. (2004) & fMRI & Semantic decision & Associative decisions task & Ambiguous > Unambiguous words & Written words & Whole brain \\
\hline Chen et al. (2008) & fMRI & Metaphors/idioms & Sentence plausibility judgment & Metaphorical > Literal meaning & Written sentences & Whole brain \\
\hline Collette et al. (2001) & PET & Verbal production & Hayling sentence completion task & Nonsalient $>$ Salient completion & Written sentences & Whole brain \\
\hline $\begin{array}{l}\text { de Zubicaray, Zelaya, Andrew, Williams, } \\
\text { \& Bullmore (2000) }\end{array}$ & fMRI & Verbal production & Hayling task variant & Nonsalient $>$ Salient completion & Written words & Whole brain \\
\hline $\begin{array}{l}\text { de Zubicaray, Wilson, McMahon, \& } \\
\text { Muthiah (2001) }\end{array}$ & fMRI & Verbal production & Picture-word interference task & Semantically related $>$ Unrelated distractors & Pictures + words & Whole brain \\
\hline $\begin{array}{l}\text { de Zubicaray, McMahon, Eastburn, \& } \\
\text { Pringle (2006) }\end{array}$ & fMRI & Verbal production & Competitor priming paradigm & Competitor Primed $>$ Unprimed & Pictures + words & ROI \\
\hline $\begin{array}{l}\text { Dobbins, Schnyer, Verfaellie, \& } \\
\text { Schacter (2004) }\end{array}$ & fMRI & Semantic decision & Categorization by features & Shift cue $>$ Consistent cue dimension & Pictures + words & ROI \\
\hline Eviatar and Just (2006) & fMRI & Metaphors/idioms & Sentence comprehension & Metaphor $>$ Literal Meaning & Written sentences & ROI \\
\hline Gennari et al. (2007) & fMRI & Homonyms & Associative decisions task & Ambiguous > Unambiguous words & Written words & ROI \\
\hline Gurd et al. (2002) & fMRI & Verbal production & Verbal fluency & Category $>$ Rote (days of the week, months) & Spoken words & Whole brain \\
\hline Gurd et al. (2002) & fMRI & Verbal production & Verbal fluency & Switching $>$ Free generation & Spoken words & Whole brain \\
\hline Hirshorn \& Thompson-Schill (2006) & fMRI & Verbal production & Verbal fluency & Switching $>$ Free generation & Written words & Whole brain \\
\hline Hirshorn \& Thompson-Schill (2006) & fMRI & Verbal production & Verbal fluency & Switching $>$ Free generation & Written words & Whole brain \\
\hline Hirshorn \& Thompson-Schill (2006) & fMRI & Verbal production & Verbal fluency & Switching $>$ Clustering & Written words & Whole brain \\
\hline
\end{tabular}




\begin{tabular}{|c|c|c|c|c|c|c|}
\hline $\begin{array}{r}\text { Hocking, McMahon, \& } \\
\text { de Zubicaray (2009) }\end{array}$ & fMRI & Verbal production & Picture naming & Semantically related $>$ Unrelated blocks & Pictures & ROI \\
\hline Hoenig and Scheef (2009) & fMRI & Homonyms & Semantic relatedness judgment & Ambiguous > Unambiguous sentences & Written sentences & Whole brain \\
\hline $\begin{array}{l}\text { Ketteler, Kastrau, Vohn, } \\
\quad \text { \& Huber (2008) }\end{array}$ & fMRI & Homonyms & Semantic relatedness judgment & Ambiguous > Unambiguous words & Written words & Whole brain \\
\hline Lee and Dapretto (2006) & fMRI & Metaphors/idioms & Semantic relatedness judgment & Metaphorical > Literal meaning & Spoken words & Whole brain \\
\hline Liu et al. (2009) & fMRI & Semantic decision & Semantic relatedness judgment & Weak $>$ Strong probe-target association & Written words & ROI \\
\hline Marshal et al. (2009) & fMRI & Metaphors/idioms & Sentence comprehension & Metaphorical > Nonsensical sentences & Written sentences & Whole brain \\
\hline Marshal et al. (2009) & fMRI & Metaphors/idioms & Sentence comprehension & Metaphorical Conjunction & Written sentences & Whole brain \\
\hline Mason and Just (2007) & fMRI & Homonyms & Sentence comprehension & Ambiguous > Unambiguous sentences & Written sentences & Whole brain \\
\hline Mason and Just (2007) & fMRI & Homonyms & Sentence comprehension & Biased ambiguous $>$ Unambiguous sentences & Written sentences & Whole brain \\
\hline Mason and Just (2007) & fMRI & Homonyms & Sentence comprehension & Biased ambiguous $>$ Balanced ambiguous & Written sentences & Whole brain \\
\hline Moss et al. (2005) & fMRI & Verbal production & Competitor priming paradigm & Competitor Primed $>$ Unprimed & Written sentences & ROI \\
\hline Nagel et al. (2008) & fMRI & Verbal production & Verb generation & High $>$ Low selection & Written words & ROI \\
\hline $\begin{array}{l}\text { Nelson, Reuter-Lorenz, Persson, } \\
\text { Sylvester, \& Johides (2009) }\end{array}$ & fMRI & Verbal production & Verb generation & High $>$ Low selection & Written words & Whole brain \\
\hline Noppeney \& Price (2002) & PET & Semantic decision & Semantic categorization & Semantic decisions conjunction & Spoken words & Whole brain \\
\hline Noppeney et al. (2004) & fMRI & Semantic decision & Synonym judgment & Difficult $>$ Easy decisions & Written words & Whole brain \\
\hline $\begin{array}{l}\text { Ochsner, Hughes, Robertson, } \\
\text { Cooper, \& Gabrieli (2009) }\end{array}$ & fMRI & Semantic decision & Eriksen flanker task & Incongruent $>$ Congruent & Written words & Whole brain \\
\hline Peelle, Troiani, \& Grossman (2009) & fMRI & Semantic decision & Sequential feature verification & Inconsistent $>$ Consistent preceding features & Written words & ROI \\
\hline Persson et al. (2004) & fMRI & Verbal production & Verb generation & High $>$ Low selection & Written words & ROI \\
\hline Race, Shanker, \& Wagner (2009) & fMRI & Semantic decision & Attribute judgment (small/organic) & Different attribute $>$ Same attribute & Written words & ROI \\
\hline Rapp et al. (2004) & fMRI & Metaphors/idioms & Sentence comprehension & Metaphorical > Literal meaning & Written sentences & Whole brain \\
\hline Rodd et al. (2005) & fMRI & Homonyms & Semantic relatedness judgment & Ambiguous > Unambiguous sentences & Auditory sentences & Whole brain \\
\hline Rodd et al. (2005) & fMRI & Homonyms & Semantic relatedness judgment & Ambiguous > Unambiguous sentences (2) & Auditory sentences & ROI \\
\hline $\begin{array}{l}\text { Roskies, Fiez, Balota, Raichle, \& } \\
\text { Petersen (2001) }\end{array}$ & PET & Semantic decision & Semantic relatedness judgment & Hard $>$ Easy decisions & Written words & ROI \\
\hline Sharp, Scott, \& Wise (2004) & PET & Semantic decision & Semantic relatedness judgment & Distorted speech $>$ Clear speech & Spoken words & ROI \\
\hline Shibata et al. (2007) & fMRI & Metaphors/idioms & Sentence comprehension & Metaphorical > Literal meaning & Written sentences & Whole brain \\
\hline $\begin{array}{l}\text { Snyder, Feigenson, \& Thompson-Schill } \\
\quad(2007)\end{array}$ & fMRI & Semantic decision & Semantic similarity judgment & Specific $>$ Global decision & Written words & Whole brain \\
\hline Spalek \& Thompson-Schill (2008) & fMRI & Verbal production & Picture-word interference task & Semantically related $>$ Unrelated distractors & Pictures and Written words & ROI \\
\hline Stringaris et al. (2007) & fMRI & Metaphors/idioms & Sentence comprehension & Metaphor > Literal Meaning & Written sentences & Whole brain \\
\hline
\end{tabular}


APPENDIX 1. (continued)

\begin{tabular}{|c|c|c|c|c|c|c|}
\hline Authors & Imaging Type & Analysis Category & Task & Contrast & Modality & Coverage \\
\hline Thompson-Schill et al. (1997) & fMRI & Verbal production & Verb generation & High $>$ Low selection & Written words & Whole brain \\
\hline Thompson-Schill et al. (1997) & fMRI & Semantic decision & Semantic classification & High $>$ Low selection & Pictures and written words & Whole brain \\
\hline Thompson-Schill et al. (1997) & fMRI & Semantic decision & Semantic comparison & High $>$ Low selection & Written words & Whole brain \\
\hline $\begin{array}{l}\text { Thompson-Schill, D’Esposito, } \\
\text { \& Kan (1999) }\end{array}$ & fMRI & Semantic decision & Attribute judgment (color/action) & Different attribute $>$ Same attribute & Written words & ROI \\
\hline Tremblay \& Gracco (2006) & fMRI & Verbal production & Verbal fluency & Unconstrained $>$ Constrained Fluency & Written words & ROI \\
\hline Wagner et al. (2001) & fMRI & Semantic decision & Semantic relatedness judgment & Four $>$ Two word choices & Written words & Whole brain \\
\hline Wagner et al. (2001) & fMRI & Semantic decision & Semantic relatedness judgment & Weak $>$ Strong probe-target association & Written words & Whole brain \\
\hline Whitney, Grossman, \& Kircher (2009) & fMRI & Homonyms & Semantic relatedness judgment & Ambiguous > Unambiguous words & Written words & Whole brain \\
\hline Whitney et al. (2009) & fMRI & Homonyms & Semantic relatedness judgment & Subordinate $>$ Dominant means & Written words & Whole brain \\
\hline Wig, Buckner, \& Schacter (2009) & fMRI & Semantic decision & Semantic comparison & Different $>$ Same comparison & Pictures + words & ROI \\
\hline $\begin{array}{l}\text { Zemplini, Haverkort, Renken, } \\
\text { \& Stowe (2007) }\end{array}$ & fMRI & Homonyms & Sentence comprehension & Ambiguous $>$ Unambiguous sentences & Written sentences & Whole brain \\
\hline $\begin{array}{l}\text { Zemplini, Renken, Hoeks, Hoogduin, } \\
\quad \text { \& Stowe (2007) }\end{array}$ & fMRI & Metaphors/idioms & Sentence comprehension & Figurative $>$ Literal Meaning & Written sentences & Whole brain \\
\hline Zhang et al. (2004) & fMRI & Semantic decision & Associative decisions task (reversible words) & High $>$ Low conflict trials & Written words & Whole brain \\
\hline
\end{tabular}

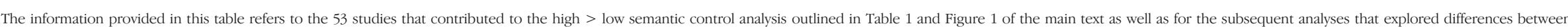

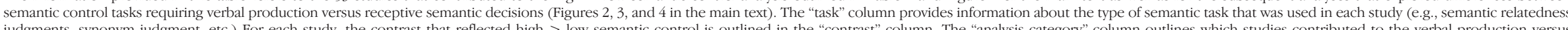

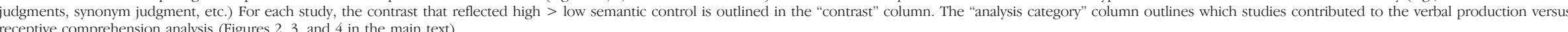


APPENDIX 2. Studies Contributing to AG (A) and pMTG (B) Clusters in the Semantic High > Low Control Analysis

Talairach

Coordinates

\begin{tabular}{|c|c|c|c|c|c|}
\hline & & & & & \\
\hline Authors & $x$ & $y$ & $z$ & Brodmann's Areas & Anaton \\
\hline$A$ & & & & & \\
\hline Assaf et al. (2006) & -39 & -50 & 44 & BA 39 & Angular \\
\hline Bedny et al. (2008) & -43 & -59 & 47 & BA 39 & Angular \\
\hline Chen et al. (2008) & -42 & -57 & 33 & BA 39 & Angular \\
\hline Gennari et al. (2007) & -34 & -46 & 43 & BA 40 & Supramarginal \\
\hline Hirshorn et al. (2006) & -55 & -59 & 46 & BA 39 & Angular \\
\hline Hirshorn et al. (2006) & -38 & -66 & 32 & BA 39 & Angular \\
\hline Hoenig and Scheef (2009) & -44 & -63 & 32 & BA 39 & Angular \\
\hline Lee and Dapretto (2006) & -46 & -60 & 48 & BA 39 & Angular \\
\hline Nagel et al. (2008) & -44 & -42 & 41 & BA 40 & Supramarginal \\
\hline Ochsner et al. (2009) & -39 & -67 & 42 & $\mathrm{BA} 7$ & Superior parietal \\
\hline Ochsner et al. (2009) & -31 & -66 & 30 & BA 18 & Visual cortex \\
\hline Peelle et al. (2009) & -42 & -66 & 24 & BA 39 & Angular \\
\hline Peelle et al. (2009) & -40 & -68 & 24 & BA 39 & Angular \\
\hline Shibata et al. (2007) & -46 & -50 & 39 & BA 39 & Angular \\
\hline Snyder et al. (2007) & -38 & -48 & 45 & BA 40 & Supramarginal \\
\hline Thompson-Schill et al. (1997) & -34 & -68 & 45 & BA 7 & Superior parietal \\
\hline
\end{tabular}

$B$

Assaf et al. (2006)

Assaf et al. (2006)

Badre et al. (2005)

Badre et al. (2005)

Bedny et al. (2008)

Boulenger et al. (2009)

Chen et al. (2008)

de Zubicaray et al. (2006)

Eviatar and Just (2006)

Gennari et al. (2007)

Ketteler et al. (2008)

Marshal et al. (2009)

Noppeney et al. (2004)

Peelle et al. (2009)

Persson et al. (2004)

$\begin{array}{rrrl}-62 & -50 & -5 & \text { BA 37, BA 21 } \\ -62 & -52 & -5 & \text { BA 37 } \\ -46 & -47 & 2 & \text { BA 21 } \\ -46 & -47 & 2 & \text { BA 21 } \\ -54 & -40 & -3 & \text { BA 21 } \\ -59 & -54 & 4 & \text { BA 37, BA 21 } \\ -62 & -38 & 7 & \text { BA 22 } \\ -57 & -44 & 5 & \text { BA 21 } \\ -49 & -52 & 7 & \text { BA 37, BA 21 } \\ -49 & -59 & 2 & \text { BA 37 } \\ -62 & -41 & -6 & \text { BA 20, BA 21 } \\ -54 & -41 & -1 & \text { BA 21 } \\ -54 & -60 & -3 & \text { BA 21 } \\ -45 & -36 & -7 & \text { BA 20, BA 21 } \\ -49 & -50 & -6 & \text { BA 20, BA 37 } \\ -53 & -58 & -2 & \text { BA 37 } \\ -47 & -45 & -10 & \text { BA 20 } \\ -53 & -54 & -2 & \text { BA 37, BA 21 }\end{array}$

Posterior occipitotemporal cortex/pMTG

Posterior occipitotemporal cortex

pMTG

pMTG

pMTG

Posterior occipitotemporal cortex/pMTG

Superior temporal gyrus

pMTG

Posterior occipitotemporal cortex/pMTG

Posterior occipitotemporal cortex

Inferior temporal gyrus/pMTG

pMTG

pMTG

Inferior temporal gyrus/pMTG

Inferior temporal gyrus/posterior occipitotemporal cortex

Rapp et al. (2004)

Rodd et al. (2005)

BA 37, BA 21

Posterior occipitotemporal cortex

Inferior temporal gyrus

Rodd et al. (2005)

Posterior occipitotemporal cortex/pMTG 
APPENDIX 2. (continued)

\begin{tabular}{|c|c|c|c|c|c|}
\hline \multirow[b]{2}{*}{ Authors } & \multicolumn{3}{|c|}{$\begin{array}{c}\text { Talairach } \\
\text { Coordinates }\end{array}$} & \multirow[b]{2}{*}{ Brodmann's Areas } & \multirow[b]{2}{*}{ Anatomical Regions } \\
\hline & $x$ & $y$ & $z$ & & \\
\hline Rodd et al. (2005) & -60 & -46 & -3 & BA 21, BA 37 & pMTG/posterior occipitotemporal cortex \\
\hline Snyder et al. (2007) & -54 & -46 & -4 & BA 20, BA 21 & Inferior temporal gyrus/pMTG \\
\hline Thompson-Schill et al. (1997) & -49 & -53 & 0 & BA 37, BA 21 & Posterior occipitotemporal cortex/pMTG \\
\hline Thompson-Schill et al. (1997) & -49 & -56 & -8 & BA 37 & Posterior occipitotemporal cortex \\
\hline Wagner et al. (2001) & -54 & -49 & -1 & BA 21 & pMTG \\
\hline Wagner et al. (2001) & -60 & -50 & 4 & BA 21 & pMTG \\
\hline Zemplini et al. (2007a) & -47 & -45 & -12 & BA 20 & Inferior temporal gyrus \\
\hline Zemplini et al. (2007a) & -57 & -41 & -6 & BA 20, BA 21 & Inferior temporal gyrus/pMTG \\
\hline Zemplini et al. (2007a) & -57 & -33 & -8 & BA 20, BA 21 & Inferior temporal gyrus/pMTG \\
\hline
\end{tabular}

A provides a list of studies contributing to the AG cluster, and B provides data for pMTG. Contributing peaks are presented in Talairach coordinates. Brodmann/anatomical labels were derived from manual inspection of each set of coordinates using MRIcron.

\section{Acknowledgments}

The work was supported by MRC program grants (MR/J004146/1) and an MRC studentship awarded to K. A. N.

Reprint requests should be sent to Prof. Matthew A. Lambon Ralph, Neuroscience and Aphasia Research Unit (NARU), Zochonis Building, School of Psychological Sciences, University of Manchester, Oxford Road, Manchester, M13 9PL, UK, or via e-mail: matt.lambon-ralph@manchester.ac.uk, Web: www.psychsci.manchester.ac.uk/naru.

\section{REFERENCES}

Allen, P., Mechelli, A., Stephan, K. E., Day, F., Dalton, J., Williams, S., et al. (2008). Fronto-temporal interactions during overt verbal initiation and suppression? Journal of Cognitive Neuroscience, 20, 1656-1669.

Amunts, K., Weiss, P. H., Mohlberg, H., Pieperhoff, P., Eickhoff, S. B., Gurd, J. M., et al. (2004). Analysis of neural mechanisms underlying verbal fluency in cytoarchitectonically defined stereotaxic space-The roles of Brodmann areas 44 and 45. Neuroimage, 22, 42-56.

Assaf, M., Calhoun, V. D., Kuzu, C. H., Kraut, M. A., Rivkin, P. R., Hart, J., et al. (2006). Neural correlates of the object recall process in semantic memory. Psychiatry Research Neuroimaging, 147, 115-126.

Badre, D., \& D'Esposito, M. (2009). Is the rostro-caudal axis of the frontal lobe hierarchical? Nature Reviews Neuroscience, 10, 659-669.

Badre, D., Poldrack, R. A., Pare-Blagoev, E. J., Insler, R. Z., \& Wagner, A. D. (2005). Dissociable controlled retrieval and generalised selection mechanisms in ventrolateral prefrontal cortex. Neuron, 47, 907-918.

Badre, D., \& Wagner, A. D. (2002). Semantic retrieval, mnemonic control, and prefrontal cortex. Behavioral and Cognitive Neuroscience Reviews, 1, 206-218.

Badre, D., \& Wagner, A. D. (2005). Frontal lobe mechanisms that resolve proactive interference. Cerebral Cortex, 15, 2003-2012.

Badre, D., \& Wagner, A. D. (2007). Left ventrolateral prefrontal cortex and the cognitive control of memory. Neuropsychologia, 45, 2883-2901.
Barbey, A. K., Colom, R., Solomon, J., Krueger, F., Forbes, C., \& Grafman, J. (2012). An integrative architecture for general intelligence and executive function revealed by lesion mapping. Brain, 135, 1154-1164.

Bedny, M., McGill, M., \& Thompson-Schill, S. L. (2008). Semantic adaptation and competition during word comprehension. Cerebral Cortex, 18, 2574-2585.

Berthier, M. L. (2001). Unexpected brain-language relationships in aphasia: Evidence from transcortical sensory aphasia associated with frontal lobe lesions. Aphasiology, 15, 99-130.

Binder, J. R., Desai, R. H., Graves, W. W., \& Conant, L. (2009). Where is the semantic system? A critical review and meta-analysis of 120 functional neuroimaging studies. Cerebral Cortex, 19, 2767-2796.

Binder, J. R., Frost, J. A., Hammeke, T. A., Bellgowan, P. S., Rao, S. M., \& Cox, R. W. (1999). Conceptual processing during the conscious resting state: A functional MRI study. Journal of Cognitive Neuroscience, 11, 80-95.

Binder, J. R., Westbury, C. F., McKiernan, K. A., Possing, E. T., \& Medler, D. A. (2005). Distinct brain systems for processing concrete and abstract concepts. Journal of Cognitive Neuroscience, 17, 905-917.

Binney, R. J., Embleton, K. V., Jefferies, E., Parker, G. J. M., \& Lambon Ralph, M. A. (2010). The ventral and inferolateral aspects of the anterior temporal lobe are crucial in semantic memory: Evidence from a novel direct comparison of distortion-corrected fMRI, rTMS, and semantic dementia. Cerebral Cortex, 20, 2728-2738.

Bottini, G., Corcoran, R., Sterzi, R., Paulesu, E., Schenone, P., Scarpa, P., et al. (1994). The role of the right hemisphere in the interpretation of figurative aspects of language: A positron emission tomography activation study. Brain, 117, 1241-1253.

Boulenger, V., Hauk, O., \& Pulvermuller, F. (2009). Grasping ideas with the motor system: Semantic somatotopy in idiom comprehension. Cerebral Cortex, 19, 1905-1914.

Brass, M., Derrfuss, J., Forstmann, B., \& Cramon, D. Y. (2005). The role of the inferior frontal junction area in cognitive control. Trends in Cognitive Sciences, 9, 314-316.

Bunge, S. A., Wendelken, C., Badre, D., \& Wagner, A. D. (2005). Analogical reasoning and prefrontal cortex: Evidence for 
separable retrieval and integration mechanisms. Cerebral Cortex, 15, 239-249.

Cabeza, R., Ciaramelli, E., \& Moscovitch, M. (2012a). Cognitive contributions of the ventral parietal cortex: An integrative theoretical account. Trends in Cognitive Sciences, 16, 338-352.

Cabeza, R., Ciaramelli, E., \& Moscovitch, M. (2012b). Response to Nelson et al.: Ventral parietal subdivisions are not incompatible with an overarching function. Trends in Cognitive Sciences, 16, 400-401.

Campanella, F., Mondani, M., Skrap, M., \& Shallice, T. (2009). Semantic access dysphasia resulting from left temporal lobe tumours. Brain, 132, 87-102.

Caspers, S., Eickhoff, S., Geyer, S., Scheperjans, F., Mohlberg, H., Zilles, K., et al. (2008). The human inferior parietal lobule in stereotaxic space. Brain Structure and Function, 212, 481-495.

Catani, M., Jones, D. K., \& Ffytche, D. H. (2005). Perisylvian language networks of the human brain. Annals of Neurology, 57, 8-16.

Chan, A. H. D., Liu, H., Yip, V., Fox, P. T., Goa, J., \& Hai Tan, L. (2004). Neural systems for word meaning modulated by semantic ambiguity. Neuroimage, 22, 1128-1133.

Chao, L. L., Haxby, J. V., \& Martin, A. (1999). Attribute-based neural substrates in temporal cortex for perceiving and knowing about objects. Nature Neuroscience, 2, 913-919.

Chen, E., Widick, P., \& Chatterjee, A. (2008).

Functional-anatomical organization of predicate metaphor processing. Brain and Language, 107, 194-202.

Chertkow, H., Bub, D., Deaudon, C., \& Whitehead, V. (1997). On the status of object concepts in aphasia. Brain and Language, 58, 203-232.

Collette, F., Hogge, M., Salmon, E., \& Van der Linden, M. (2006). Exploration of the neural substrate of executive functioning by functional imaging. Neuroscience, 139, 209-221.

Collette, F., Van der Linden, M., Delfiore, G., Degueldre, C., Luxen, A., \& Salmon, E. (2001). The functional anatomy of inhibition processes investigated with the hayling task. NeuroImage, 14, 258-267.

Corbett, F., Jefferies, E., Ehsan, S., \& Lambon Ralph, M. A. (2009). Different impairments of semantic cognition in semantic dementia and semantic aphasia: Evidence from the non-verbal domain. Brain, 132, 2593-2608.

Corbett, F., Jefferies, E., \& Lambon Ralph, M. A. (2009). Exploring multimodal semantic control impairments in semantic aphasia: Evidence from naturalistic object use. Neuropsychologia, 47, 2721-2731.

Corbett, F., Jefferies, E., \& Lambon Ralph, M. A. (2011). Deregulated semantic cognition follows prefrontal and temporo-parietal damage: Evidence from the impact of task constraint on nonverbal object use. Journal of Cognitive Neuroscience, 23, 1125-1135.

Cristescu, T. C., Devlin, J. T., \& Nobre, A. C. (2006). Orienting attention to semantic categories. Neuroimage, 33, 1178-1187.

de Zubicaray, G. I., McMahon, K. L., Eastburn, M., \& Pringle, A. (2006). Top-down influences on lexical selection during spoken word production: A 4T fMRI investigation of refractory effects in picture naming. Human Brain Mapping, 27, 864-873.

de Zubicaray, G. I., Wilson, S. J., McMahon, K. L., \& Muthiah, S. (2001). The semantic interference effect in the picture-word paradigm: An event-related fMRI study employing overt responses. Human Brain Mapping, 14, 218-227.

de Zubicaray, G. I., Zeleya, F. O., Andrew, C., Williams, S., \& Bullmore, E. T. (2000). Cerebral regions associated with verbal response initiation, suppression and strategy use. Neuropsychologia, 38, 1292-1304.

Demb, J. B., Desmond, J. E., Wagner, A. D., Vaidya, C. J., Glover, G. H., \& Gabrieli, J. D. (1995). Semantic encoding and retrieval in the left inferior prefrontal cortex: A functional MRI study of task difficulty and process specificity. Journal of Neuroscience, 15, 5870-5878.

Devlin, J. T., Matthews, P. M., \& Rushworth, M. F. S. (2003). Semantic processing in the left inferior prefrontal cortex: A combined functional magnetic resonance imaging and transcranial magnetic stimulation study. Journal of Cognitive Neuroscience, 15, 71-84.

Dobbins, I. G., Schnyer, D. M., Verfaellie, M., \& Schacter, D. L. (2004). Cortical activity reductions during repetition priming can result from rapid response learning. Nature, $428,316-319$.

Duncan, J. (2006). Brain mechanisms of attention. The Quarterly Journal of Experimental Psychology, 59, 2-27.

Duncan, J. (2010). The multiple-demand (MD) system of the primate brain: Mental programs for intelligent behaviour. Trends in Cognitive Sciences, 14, 172-179.

Duncan, J., \& Owen, A. M. (2000). Common regions of the human frontal lobe recruited by diverse cognitive demands. Trends in Neuroscience, 23, 475-483.

Eviatar, Z., \& Just, M. A. (2006). Brain correlates of discourse processing: An fMRI investigation of irony and conventional metaphor comprehension. Neuropsychologia, 44, 2348-2359.

Friederici, A. D. (2009). Pathways to language: Fiber tracts in the human brain. Trends in Cognitive Sciences, 13, 175-181.

Gabrieli, J. D. E., Poldrack, R. A., \& Desmond, J. E. (1998). The role of left prefrontal cortex in language and memory. Proceedings of the National Academy of Sciences, U.S.A., 95, 906-913.

Gainotti, G. (2000). What the locus of brain lesion tells us about the nature of the cognitive defect underlying category-specific disorders: A review. Cortex, 36, 539-559.

Gardner, H. E., Lambon Ralph, M. A., Dodds, N., Jones, T., Eshan, S., \& Jefferies, E. (2012). The differential contributions of prefrontal and temporoparietal cortices to multimodal semantic control: Exploring refractory effects in semantic aphasia. Journal of Cognitive Neuroscience, 24, 778-793.

Gennari, S. P., MacDonald, M. C., Postle, B. R., \& Seidenberg, M. S. (2007). Context-dependent interpretation of words: Evidence for interactive neural processes. Neuroimage, 35, 1278-1286.

Gerlach, C. (2007). A review of functional imaging studies on category specificity. Journal of Cognitive Neuroscience, 19, 296-314

Gold, B. T., Balota, D. A., Jones, S. J., Powell, D. K., Smith, C. D., \& Andersen, A. H. (2006). Dissociation of automatic and strategic lexical-semantics: Functional magnetic resonance imaging evidence for differing roles of multiple frontotemporal regions. Journal of Neuroscience, 26, 6523-6532.

Gold, B. T., \& Buckner, R. L. (2002). Common prefrontal regions coactivate with dissociable posterior regions during controlled semantic and phonological tasks. Neuron, 35, 803-812.

Gough, P. M., Nobre, A. C., \& Devlin, J. T. (2005). Dissociating linguistic processes in the left inferior frontal cortex with transcranial magnetic stimulation. Journal of Neuroscience, 25, 8010-8016.

Gurd, J. M., Amunts, K., Weiss, P. H., Zafiris, O., Zilles, K., Marshall, J. C., et al. (2002). Brain correlates of discourse processing: An fMRI investigation of irony and conventional metaphor comprehension. Brain, 125, 1024-1038. 
Hart, J., \& Gordon, B. (1990). Delineation of single-word semantic comprehension deficits in aphasia, with anatomic correlation. Annals of Neurology, 27, 226-231.

Head, H. (1926). Aphasia and kindred disorders of speech. London: Cambridge University Press.

Hickok, G., \& Poeppel, D. (2004). Dorsal and ventral streams: A framework for understanding aspects of the functional anatomy of language. Cognition, 92, 67-99.

Hickok, G., \& Poeppel, D. (2007). The cortical organization of speech processing. Nature Reviews Neuroscience, 8 , 393-402.

Hillis, A., \& Caramazza, A. (1991). Category-specific naming and comprehension impairment: A double dissociation. Brain, 114, 2081-2094.

Hirshorn, E. A., \& Thompson-Schill, S. L. (2006). Role of the left inferior frontal gyrus in covert word retrieval: Neural correlates of switching during verbal fluency. Neuropsychologia, 44, 2547-2557.

Hocking, J., McMahon, K. L., \& de Zubicaray, G. I. (2009). Semantic context and visual feature effects in object naming: An fMRI study using arterial spin labeling. Journal of Cognitive Neuroscience, 21, 1571-1583.

Hoenig, K., \& Scheef, L. (2009). Neural correlates of semantic ambiguity processing during context verification. Neuroimage, 45, 1009-1019.

Hoffman, P., Jefferies, E., \& Lambon Ralph, M. A. (2010). The role of ventrolateral prefrontal cortex in abstract word comprehension: Convergent neuropsychological and rTMS evidence. Journal of Neuroscience, 30, 15450-15456.

Hoffman, P., Pobric, G., Drakesmith, M., \& Lambon Ralph, M. A. (2011). Posterior middle temporal gyrus is involved in verbal and non-verbal semantic cognition: Evidence from rTMS. Aphasiology, 26, 1119-1130.

Hutchinson, J. B., Uncapher, M. R., Weiner, K. S., Bressler, D. W., Silver, M. A., Preston, A. R., et al. (2012). Functional heterogeneity in posterior parietal cortex across attention and episodic memory retrieval. Cerebral Cortex. doi: 10.1093/cercor/bhs278.

Jefferies, E. (2013). The neural basis of semantic cognition: Converging evidence from neuropsychology, neuroimaging and TMS. Cortex, 49, 611-625.

Jefferies, E., Baker, S. S., Doran, M., \& Lambon Ralph, M. A. (2007). Refractory effects in stroke aphasia: A consequence of poor semantic control. Neuropsychologia, 45, 1065-1079.

Jefferies, E., \& Lambon Ralph, M. A. (2006). Semantic impairment in stroke aphasia versus semantic dementia: A case-series comparison. Brain, 129, 2132-2147.

Joseph, J. E. (2001). Functional neuroimaging studies of category specificity in object recognition: A critical review and meta-analysis. Cognitive, Affective, \& Behavioral Neuroscience, 1, 119-136.

Kable, J. W., Kan, I. P., Wilson, A., Thompson-Schill, S. L., \& Chatterjee, A. (2005). Conceptual representations of action in the lateral temporal cortex. Journal of Cognitive Neuroscience, 17, 1855-1870.

Kemmerer, D., Rudrauf, D., Manzel, K., \& Tranel, D. (2012). Behavioral patterns and lesion sites associated with impaired processing of lexical and conceptual knowledge of actions. Cortex, 48, 826-848.

Kertesz, A., Sheppard, A., \& MacKenzie, R. (1982). Localisation in trasncortical sensory aphasia. Archives of Neurology, 39, 475-478.

Ketteler, D., Kastrau, F., Vohn, R., \& Huber, W. (2008). The subcortical role of language processing. High level linguistic features such as ambiguity-resolution and the human brain; an fMRI study. Neuroimage, 39, 2002-2009.

Laird, A. R., Fox, P. M., Price, C., Glahn, D. C., Uecker, A. M., Lancester, J. L., et al. (2005). ALE meta-analysis: Controlling the false discovery rate and performing statistical contrasts Human Brain Mapping, 25, 155-164.

Lambon Ralph, M. A., Pobric, G., \& Jefferies, E. (2009). Conceptual knowledge is underpinned by the temporal pole bilaterally: Convergent evidence from rTMS. Cerebral Cortex, 19, 832-838.

Lambon Ralph, M. A., Sage, K., Jones, R., \& Mayberry, E. J. (2010). Coherent concepts are computed in the anterior temporal lobes. Proceedings of the National Academy of Sciences, U.S.A., 107, 2717-2722.

Lee, S. S., \& Dapretto, M. (2006). Metaphorical vs. literal word meanings: fMRI evidence against a selective role of the right hemisphere. Neuroimage, 29, 536-544.

Lerner, Y., Honey, C. J., Silbert, L. J., \& Hasson, U. (2011). Topographic mapping of a hierarchy of temporal receptive windows using a narrated story. The Journal of Neuroscience, 31, 2906-2915.

Liu, L., Deng, X., Peng, D., Cao, F., Ding, G., Jin, Z., et al. (2009). Modality- and task-specific brain regions involved in Chinese lexical processing. Journal of Cognitive Neuroscience, 21, $1473-1487$

Luria, A. R. (1976). The working brain: An introduction to neuropsychology. London: Penguin.

Martin, A. (2007). The representation of object concepts in the brain. Annual Review of Psychology, 58, 25-45.

Mashal, N., Faust, M., Hendler, T., \& Jung-Beeman, M. (2009). An fMRI study of processing novel metaphoric sentences. Laterality: Asymmetries of Body, Brain and Cognition, 14, 30-54.

Mason, R. A., \& Just, M. A. (2007). Lexical ambiguity in sentence comprehension. Brain Research, 1146, 115-127.

Mayberry, E. J., Sage, K., \& Lambon Ralph, M. A. (2011). At the edge of semantic space: The breakdown of coherent concepts in semantic dementia is constrained by typicality and severity but not modality. Journal of Cognitive Neuroscience, 23, 2240-2251.

McClelland, J. L., \& Rogers, T. T. (2003). The parallel distributed processing approach to semantic cognition. Nature Reviews Neuroscience, 4, 310-322.

Metzler, C. (2001). Effects of frontal lobe lesions on the slection of context appropriate meanings. Neuropsychology, 15, 315-328.

Mion, M., Patterson, K., Acosta-Cabronero, J., Pengas, G., Izquierdo-Garcia, D., Hong, Y. T., et al. (2010). What the left and right anterior fusiform gyri tell us about semantic memory. Brain, 133, 3256-3268.

Moss, H. E., Abdallah, S., Fletcher, P., Bright, P., Pilgrim, L., Acres, K., et al. (2005). Selecting among competing alternatives: Selection and retrieval in the left inferior frontal gyrus. Cerebral Cortex, 15, 1723-1735.

Nagel, I. E., Schumacher, E. H., Goebel, R., \& D'Esposito, M. (2008). Functional MRI investigation of verbal selection mechanisms in lateral prefrontal cortex. Neuroimage, 43, 801-807.

Nee, D. E., Wager, T. D., \& Johides, J. (2007). Interference resolution: Insights from a meta-analysis of neuroimaging tasks. Cognitive Affective \& Behavioural Neuroscience, 7 , $1-17$.

Nelson, S. M., McDermott, K. B., \& Petersen, S. E. (2012). In favor of a "fractionation" view of ventral parietal cortex: Comment on Cabeza et al. Trends in Cognitive Sciences, 16, 399-400.

Nelson, J. K., Reuter-Lorenz, P. A., Persson, J., Sylvester, C. Y. C., \& Johides, J. (2009). Mapping interference resolution across task domains: A shared control process in left inferior frontal gyrus. Brain Research, 1256, 92-100.

Noonan, K., Jefferies, E., Corbett, F., \& Lambon Ralph, M. A. (2010). Elucidating the nature of deregulated semantic 
cognition in semantic aphasia: Evidence for the roles of prefrontal and temporoparietal cortices. Journal of Cognitive Neuroscience, 22, 1597-1613.

Noppeney, U., \& Price, C. (2002). A PET study of stimulusand task-induced semantic processing. Neuroimage, 15 , 927-935.

Noppeney, U., Phillips, J., \& Price, C. (2004). The neural areas that control the retrieval and selection of semantics. Neuropsychologia, 42, 1269-1280.

Ochsner, K. N., Hughes, B., Robertson, E. R., Cooper, J. C., \& Gabrieli, J. D. (2009). Neural systems supporting the control of affective and cognitive conflicts. Journal of Cognitive Neuroscience, 21, 1841-1854.

Patterson, K. (2007). The reign of typicality in semantic memory. Philosophical Transactions of the Royal Society of London, Series B, Biological Sciences, 362, 813-821.

Patterson, K., Nestor, P. J., \& Rogers, T. (2007). Where do you know what you know? The representation of semantic knowledge in the human brain. Nature Reviews Neuroscience, 8, 976-987.

Peelle, J. E., Troiani, V., \& Grossman, M. (2009). Interaction between process and content in semantic memory: An fMRI study of noun feature knowledge. Neuropsychologia, 47, 995-1003.

Peers, P. V., Ludwig, C. J. H., Rorden, C., Cusack, R., Bonfiglioli, C., Bundesen, C., et al. (2005). Attentional functions of parietal and frontal cortex. Cerebral Cortex, 15, 1469-1484.

Persson, J., Sylvester, C. Y. C., Nelson, J. K., Welsh, K. M., Johides, J., \& Reuter-Lorenz, P. A. (2004). Selection requirements during verb generation: Differential recruitment in older and younger adults. Neuroimage, 23, 1382-1390.

Pobric, G., Jefferies, E., \& Lambon Ralph, M. A. (2007). Anterior temporal lobes mediate semantic representation: Mimicking semantic dementia by using rTMS in normal participants. Proceedings of the National Academy of Sciences, U.S.A., 104, 20137-20141.

Pobric, G., Jefferies, E., \& Lambon Ralph, M. A. (2010). Category-specific versus category-general semantic impairment induced by transcranial magnetic stimulation. Current Biology, 20, 964-968.

Poldrack, R. A., Wagner, A. D., Prull, M. W., Desmond, J. E., Glover, G. H., \& Gabrieli, J. D. (1999). Functional specialization for semantic and phonological processing in the left inferior prefrontal cortex. Neuroimage, 10, $15-35$.

Race, E. A., Shanker, S., \& Wagner, A. D. (2009). Neural priming in human frontal cortex: Multiple forms of learning reduce demands on the prefrontal executive system. Journal of Cognitive Neuroscience, 21, 1766-1781.

Raichle, M. E., MacLeod, A. M., Snyder, A. Z., Powers, W. J., Gusnard, D. A., \& Shulman, G. L. (2001). A default mode of brain function. Proceedings of the National Academy of Sciences, U.S.A., 98, 676-682.

Rapp, A. M., Leube, D. T., Erb, M., Grodd, W., \& Kircher, T. T. J. (2004). Neural correlates of metaphor processing. Cognitive Brain Research, 20, 395-402.

Robson, H., Sage, K., \& Lambon Ralph, M. A. (2012). Wernicke's aphasia reflects a combination of acoustic-phonological and semantic control deficits: A case-series comparison of Wernicke's aphasia, semantic dementia and semantic aphasia. Neuropsychologia, 50, 266-275.

Rodd, J. M., Davis, M. H., \& Johnsrude, I. S. (2005). The neural mechanisms of speech comprehension: fMRI studies of semantic ambiguity. Cerebral Cortex, 15, 1261-1269.

Rogers, T. T., Lambon Ralph, M. A., Garrard, P., Bozeat, S., McClelland, J. L., Hodges, J. R., et al. (2004). The structure and deterioration of semantic memory: A neuropsychological and computational investigation. Psychological Review, 111, 205-235.

Roskies, A. L., Fiez, J. A., Balota, D. A., Raichle, M. E., \& Petersen, S. E. (2001). Task-dependent modulation of regions in the left inferior frontal cortex during semantic processing. Journal of Cognitive Neuroscience, 13, 829-843.

Saur, D., Kreher, B. W., Schnell, S., Kummerer, D., Kellmeyer, P., Vry, M. S., et al. (2008). Ventral and dorsal pathways for language. Proceedings of the National Academy of Sciences, U.S.A., 105, 18035-18040.

Schnur, T. T., Schwartz, M. F., Kimberg, D., Hirshorn, E., Coslett, H. B., \& Thompson-Schill, S. L. (2009). Localizing interference during naming: Convergent neuroimaging and neuropsychological evidence for the function of Broca's area. Proceedings of the National Academy of Sciences, U.S.A., 106, 322-327.

Schwartz, M., Kimberg, D. Y., Walker, G. M., Faseyitan, O., Brecher, A., Dell, G. S., et al. (2009). Anterior temporal involvement in semantic word retrieval: Voxel-based lesion-symptom mapping evidence from aphasia. Brain, 132, 3411-3427.

Seghier, M. L., Fagan, E., \& Price, C. (2010). Functional subdivisions in the left angular gyrus where the semantic system meets and diverges from the default network. Journal of Neuroscience, 30, 16809-16817.

Sharp, D. J., Awad, M., Warren, J. E., Wise, R. J. S., Vigliocco, G., $\&$ Scott, S. K. (2010). The neural response to changing semantic and perceptual complexity during language processing. Human Brain Mapping, 31, 365-377.

Sharp, D. J., Scott, S. K., \& Wise, R. (2004). Monitoring and the controlled processing of meaning: Distinct prefrontal systems. Cerebral Cortex, 14, 1-10.

Shibata, M., Abe, J., Terao, A., \& Miyamoto, T. (2007). Neural mechanisms involved in the comprehension of metaphoric and literal sentences: An fMRI study. Brain Research, 1166, 92-102.

Spalek, K., \& Thompson-Schill, S. L. (2008). Task-dependent semantic interference in language production: An fMRI study. Brain \& Language, 107, 220-228.

Spitsyna, G., Warren, J. E., Scott, S. K., Turkheimer, F. E., \& Wise, R. J. S. (2006). Converging language streams in the human temporal lobe. Journal of Neuroscience, 26, $7328-7336$

Spreng, R. N., Stevens, W. D., Chamberlain, J. P., Gilmore, A. W., \& Schacter, D. L. (2010). Default network activity, coupled with the frontoparietal control network, supports goal-directed cognition. Neuroimage, 53, 303-317.

Stringaris, A. K., Medford, N. C., Giampietro, V., Brammer, M. J., \& David, A. S. (2007). Deriving meaning: Distinct neural mechanisms for metaphoric, literal, and non-meaningful sentences. Brain and Language, 100, 150-162.

Stuss, D. T., \& Alexander, M. P. (2007). Is there a dysexecutive syndrome? Philosophical Transactions of the Royal Society of London, Series B, Biological Sciences, 362, 901-915.

Synder, H. R., Banich, M. T., \& Munakata, Y. (2011). Choosing our words: Retrieval and selection processes recruit shared neural substrates in left ventrolateral prefrontal cortex. Journal of Cognitive Neuroscience, 23, 3470-3482.

Snyder, H. R., Feigenson, K., \& Thompson-Schill, S. L. (2007). Prefrontal cortical response to conflict during semantic and phonological tasks. Journal of Cognitive Neuroscience, 19, 761-775.

Thompson-Schill, S. L. (2003). Neuroimaging studies of semantic memory: Inferring "how" from "where." Neuropsychologia, 41, 280-292.

Thompson-Schill, S. L., D'Esposito, M., Aguirre, G. K., \& Farah, M. J. (1997). Role of left inferior prefrontal cortex 
in retrieval of semantic knowledge: A reevaluation. Proceedings of the National Academy of Sciences, U.S.A., 94, 14792-14797.

Thompson-Schill, S. L., D’Esposito, M., \& Kan, I. P. (1999). Effects of repetition and competition on activity in left prefrontal cortex during word generation. Neuron, 23, 513-522.

Thompson-Schill, S. L., Swick, D., Farah, M. J., D’Esposito, M., Kan, I. P., \& Knight, R. T. (1998). Verb generation in patients with focal frontal lesions: A neuropsychological test of neuroimaging findings. Proceedings of the National Academy of Sciences, U.S.A., 95, 15855-15860.

Tremblay, P., \& Gracco, V. (2006). Contribution of the frontal lobe to externally and internally specified verbal responses: fMRI evidence. Neuroimage, 33, 947-957.

Turken, A. U., \& Dronkers, N. F. (2011). The neural architecture of the language comprehension network: Converging evidence from lesion and connectivity analyses. Frontiers in Systems Neuroscience, 5, 1-20.

Uddin, L. Q., Supekar, K., Amin, H., Rykhlevskaia, E., Nguyen, D. A., Greicius, M. D., et al. (2010). Dissociable connectivity within human angular gyrus and intraparietal sulcus: Evidence from functional and structural connectivity. Cerebral Cortex, 20, 2636-2646.

Vandenberghe, R., Price, C., Wise, R., Josephs, O., \& Frackowiak, R. S. (1996). Functional anatomy of a common semantic system for words and pictures. Nature, 383, 254-256.

Vigneau, M., Beaucousin, V., Hervé, P. Y., Duffau, H., Crivello, F., Houdé, O., et al. (2006). Meta-analyzing left hemisphere language areas: Phonology, semantics, and sentence processing. Neuroimage, 30, 1414-1432.

Vincent, J. L., Kahn, I., Snyder, A. Z., Raichle, M. E., \& Buckner, R. L. (2008). Evidence for a frontoparietal control system revealed by intrinsic functional connectivity. Journal of Neurophysiology, 100, 3328-3342.

Visser, M., Jefferies, E., Embleton, K. V., \& Lambon Ralph, M. A. (in press). Evidence for a caudo-rostral gradient of information convergence in the temporal lobes: An fMRI study of verbal and non-verbal semantic processing. Journal of Cognitive Neuroscience.

Visser, M., Jefferies, E., \& Lambon Ralph, M. A. (2010). Semantic processing in the anterior temporal lobes: A meta-analysis of the functional neuroimaging literature. Journal of Cognitive Neuroscience, 22, 1083-1094.

Visser, M., \& Lambon Ralph, M. A. (2011). Differential contributions of bilateral ventral anterior temporal lobe and left anterior superior temporal gyrus to semantic processes. Journal of Cognitive Neuroscience, 23, 3121-3131.

Wagner, A. D., Pare-Blagoev, E. J., Clark, J., \& Poldrack, R. A. (2001). Recovering meaning; Left prefrontal cortex guides controlled semantic retrieval. Neuron, 31, 329-338.

Wang, J., Conder, J., Blitzer, D., \& Shinkareva, S. (2010). Neural representation of abstract and concrete concepts:
A meta-analysis of neuroimaging studies. Human Brain Mapping, 31, 1459-1468.

Warrington, E., \& McCarthy, R. (1987). Categories of knowledge: Further fractionations and an attempted integration. Brain, 110, 1273-1296.

Warrington, E., \& McCarthy, R. (1994). Multiple meaning systems in the brain: A case for visual semantics. Neuropsychologia, 32, 1465-1473.

Warrington, E., \& Shallice, T. (1984). Category specific semantic impairments. Brain, 107, 829-854.

Whitney, C., Grossman, M., \& Kircher, T. T. J. (2009). The influence of multiple primes on bottom-up and top-down regulation during meaning retrieval: Evidence for 2 distinct neural networks. Cerebral Cortex, 19, 2548-2560.

Whitney, C., Jefferies, E., \& Kircher, T. (2010a). Heterogeneity of the left temporal lobe in semantic representation and control: Priming multiple vs. single meanings of ambiguous words. Cerebral Cortex, 21, 831-844.

Whitney, C., Jefferies, E., \& Kircher, T. (2010b). Heterogeneity of the left temporal lobe in semantic representation and control: Priming multiple vs. single meanings of ambiguous words. Cerebral Cortex, 21, 831-844.

Whitney, C., Kirk, M., O’Sullivan, J., Lambon Ralph, M. A., \& Jefferies, E. (2010). The neural organization of semantic control: TMS evidence for a distributed network in left inferior frontal and posterior middle temporal gyrus. Cerebral Cortex, 21, 1066-1075.

Whitney, C., Kirk, M., O’Sullivan, J., Lambon Ralph, M. A., \& Jefferies, E. (2012). Executive semantic processing is underpinned by a large-scale neural network: Revealing the contribution of left prefrontal, posterior temporal and parietal cortex to controlled retrieval and selection using TMS. Journal of Cognitive Neuroscience, 24, 133-147.

Wig, G. S., Buckner, R. L., \& Schacter, D. L. (2009). Repetition priming influences distinct brain systems: Evidence from task-evoked data and resting-state correlations. Journal of Neurophysiology, 101, 2632-2648.

Woolgar, A., Hampshire, A., Thompson, R., \& Duncan, J. (2011). Adaptive coding of task-relevant information in human frontoparietal cortex. Journal of Neuroscience, 31, 14592-14599.

Xiang, H. D., Fonteijn, H. M., Norris, D. G., \& Hagoort, P. (2010). Topographical functional connectivity pattern in the perisylvian language networks. Cerebral Cortex, 20, 549-560.

Zempleni, M.-Z., Haverkort, M., Renken, R., \& Stowe, L. A. (2007). Evidence for bilateral involvement in idiom comprehension: An fMRI study. Neuroimage, 34, 1280-1291.

Zempleni, M.-Z., Renken, R., Hoeks, J. C. J., Hoogduin, J. M., \& Stowe, L. A. (2007). Semantic ambiguity processing in sentence context: Evidence from event-related fMRI. Neuroimage, 34, 1270-1279.

Zhang, X. J., Zhuang, J., Ma, L., Yu, W., Peng, D., Ding, G., et al. (2004). Evidence for bilateral involvement in idiom comprehension: An fMRI study. Neuroimage, 23, 975-982. 